\title{
Sway and Disturbance Rejection Control for Varying Rope Tower Cranes Suffering from Friction and Unknown Payload Mass
}

Zheng Tian ( $\nabla$ ztian97@njtech.edu.cn )

Nanjing Tech University https://orcid.org/0000-0002-0477-9598

Lili Yu

Nanjing Tech University https://orcid.org/0000-0002-5097-2591

Huimin Ouyang

Nanjing Tech University

Guangming Zhang

Nanjing Tech University

\section{Research Article}

Keywords: Underactuated system,Tower crane, Time-varying rope length, Sway rejection, Robust adaptive sliding mode control

Posted Date: March 1st, 2021

DOl: https://doi.org/10.21203/rs.3.rs-171106/v1

License: (9) This work is licensed under a Creative Commons Attribution 4.0 International License.

Read Full License

Version of Record: A version of this preprint was published at Nonlinear Dynamics on August 12th, 2021.

See the published version at https://doi.org/10.1007/s11071-021-06793-6. 


\title{
Sway and Disturbance Rejection Control for Varying Rope Tower Cranes Suffering from Friction and Unknown Payload Mass
}

\author{
Zheng Tian · Lili Yu* ${ }^{*}$ Huimin Ouyang · Guangming Zhang
}

Received: date / Accepted: date

\begin{abstract}
Tower cranes are well-known underactuated systems, where the design of controllers for them with time-varying rope length was weak in the past because of their complex dynamic characteristic. The payload oscillation will become worse when the jib slew angle, the trolley position and the rope length are changed simultaneously. The proposed method is designed based on robust adaptive sliding mode control via tracking non-zero initial reference trajectories, in which, frictions and lumped disturbances in the crane system are eliminated, as well as unknown payload mass is effectively estimated online. Lyapunov technique is combined with LaSalle's invariance theorem to design controller and analyze stability. Various and strict simulations are applied, which validate the effectiveness and extreme robustness of the proposed method.
\end{abstract}

Keywords Underactuated system - Tower crane . Time-varying rope length · Sway rejection - Robust adaptive sliding mode control

\section{Introduction}

Because of their combination of simple structure and complex dynamic characteristics, underactuated system$\mathrm{s}$ where the number of actuators is less than the DOF (degrees of freedom) have been paid more and more attention to the control technique improvement in recent years [1]. Typical underactuated systems include underactuated manipulators [2], underactuated mobile robots [3], reaction wheel pendulums [4], and cranes [5],

Zheng Tian · Lili $\mathrm{Yu}^{*} \cdot$ Huimin Ouyang $\cdot$ Guangming Zhang College of Electrical Engineering and Control Science, Nanjing Tech University, No.30, Puzhu Road(s), Nanjing, 211816, China

E-mail: lili.yu@njtech.edu.cn etc. Among them, cranes are common and utilized to transport payload over long distances in factories, ports, construction sites, and so on.

Tower crane is a kind of crane that transports goods in 3D (three-dimension) space. Its transportation process is often accompanied by the translation of the trolley and the rotation of the jib. These two driving mechanisms with different motion properties make the dynamic model and design corresponding control method more complicated. At the same time, with the lifting movement of the payload, the length of the rope will change, which will have a great impact on the dynamic characteristics of the tower crane, such as the natural frequency of the payload swing. In addition, at the actual control applications, it is inevitable that there will be friction effect, incomplete theoretical modeling, parameter uncertainties and external disturbances of full DOF. In these cases, it is a very challenging issue to realize the accurate positioning of the jib, trolley and payload lifting with quickly suppressing the swing of the payload.

In the past, many controllers aiming at cranes to realize payload transportation and swing suppression have been worked out, which include input-shaping [6], sliding mode control [7][8], adaptive control [9]-[13], coupling based method [14]-[16], robust control [17]-[19], optimal control [20], intelligent control [21] [22] and adaptive neural network sliding mode control [23], which is control method combining intelligent algorithm and traditional controller.

However, to increase transportation efficiency, the rope is often hoisted and lowered in many actual conditions. Consequently, these above approaches that are all not mentioned the presence of the changed rope length are not certifiable the control performance and system stability. 
To solve the control problems of time-varying rope length condition for cranes, Ramli et al. presented an efficient controller using NNUMZV-APIDLNN algorithm considering payload hoisting and disturbances for overhead cranes, where significant reduction effect in the swing angles was derived with experimental results [24]. Li et al. designed a coupling-based controller for underactuated overhead cranes to anti-swing and transferring payload [25]. Finite-time flatness-based regulation controllers [26] were designed for cranes with both constant and varying cable length, and anti-swing and high-speed positioning problems were cracked owing to Zhang et al. An adaptive coupling method subject to unknown model parameters was proposed by Sun et al. and verified by experiments, which could achieve precise trolley positioning and payload hoisting/lowering control as well as fast swing elimination [27]. Lu et al. [28] presented an enhanced-coupling adaptive controller for overhead cranes, which had improvement in the control performance and increased the robustness aiming at the unknown payload mass. But, this method just solves the unknown payload mass problem, other common influence such as friction or external disturbances is omitted. In another research to solve the payload hoisting effect problem, an improved UMZV shaper by using the PSO algorithm to select the optimal control parameters was utilized for underactuated 3D overhead cranes [29]. Yang et al. [30] designed a nonlinear actuator constraints controller combining state observer and online friction compensation, which achieved good results about jib/trolley positioning and payload sway suppression by experimental validation.

After illustrating the recent existed researches, there are still some intractable problems need to be solve:

1) Traditional underactuated crane positioning and antisway control is usually aimed at overhead crane systems. Even if overhead cranes with multiple DOF move in $3 \mathrm{D}$ space, the driveable mechanism is still work in linear force, the dynamic characteristics are still simple, and the corresponding controllers design is relatively convenient. But when different driving forces appear in the crane transportation task, such as in tower crane control, one direction is the translation force of the trolley, and the other direction is the jib torque. At this time, due to the participation of eccentric motion for payload, the system dynamics becomes very complicated. At the same time, when the payload is lifted and lowered, the natural frequency of the payload swing will change. Hence, the failure of the controller designed for the ordinary single-pendulum will inevitably occur.

2) For traditional controllers, on the one hand, only positioning can be achieved under normal circum- stances, but the swing suppression effect is not good, and the overall convergence speed is slow; On the other hand, most controllers use adjustment control methods for the target position, but in practical applications, the regulation control will produce a great initial output value of the controller, causing inevitable initial fluctuations, damaging the life of drivers and affecting the effect of anti-swing.

3) In real applications, many factors, such as, the friction of input channel, unmodeled part effect, parameter uncertainties and full DOF external disturbances, are inevitably appear in motion process, while many control strategies do not fully consider and restrain their adverse effects. Under these circumstances, it is a very challenging problem to achieve accurate positioning of the actuated mechanism while quickly suppressing the swing of the payload.

The above problems are clearly stated and the main contributions of this paper are as follows:

1) Linearized model is not needed in the proposed controller, and a sliding mode surface vector with nonlinear variable gains and non-zero initial reference trajectories is designed. As long as there exists error in the system, the variable gains will continue to be changed adaptively, so that the stable convergence speed of the tower crane system is improved compared with traditional SMC methods.

2) Adaptive technology is used to suppress the influence of friction, unmodeled parts, parameter uncertainties and external disturbances, and specially, in order to solve the problem that the unknown payload mass has an impact on the rope lifting motion, the mass value is also self-adapted. Hence, the proposed controller presents both good control performance and extreme robustness in simulations.

A robust adaptive sliding mode controller is proposed in this paper. Firstly, in Section 2, the model of tower crane with time-varying rope length considering friction, model parameters uncertainties, the unconsidered nonlinear factors and external disturbances is introduced. Then, the properties of non-zero initial reference trajectories are showed. In Section 3, to improve the convergence speed of traditional sliding mode control, a sliding mode surface with variable gains is designed, and the gains is changed by error adaptively. The friction, lumped disturbance and unknown payload mass is estimated by adaptive technique. The control system stability is proved by Lyapunov technique and LaSalle's invariance principle strictly. In Section 4, many representative simulations are conducted to demonstrate its effectiveness on trajectory track- 
ing, swing elimination, friction compensation, lumped disturbances suppression and payload mass estimation. And, Section 5 is conclusion of this paper.

\section{Problem Statement}

The model diagram of tower cranes with time-varying rope length is presented as shown in Fig. 1, whose mathematical model is built by the Lagrange's kinematics equations, and the relations are as follows [31]:

$$
\begin{aligned}
& \boldsymbol{M}(\boldsymbol{q}) \ddot{\boldsymbol{q}}+\boldsymbol{C}(\boldsymbol{q}, \dot{\boldsymbol{q}}) \dot{\boldsymbol{q}}+\boldsymbol{G}(\boldsymbol{q})=\boldsymbol{U}-\boldsymbol{F}_{s}(\dot{\boldsymbol{q}})+\boldsymbol{D}, \\
& \boldsymbol{M}(\boldsymbol{q})=\left[\begin{array}{lllll}
m_{11} & m_{12} & m_{13} & m_{14} & m_{15} \\
m_{21} & m_{22} & m_{23} & m_{24} & m_{25} \\
m_{31} & m_{32} & m_{33} & m_{34} & m_{35} \\
m_{41} & m_{42} & m_{43} & m_{44} & m_{45} \\
m_{51} & m_{52} & m_{53} & m_{54} & m_{55}
\end{array}\right] \text {, } \\
& m_{11}=J_{0}+m x^{2}+M_{t} x^{2}+m l^{2}-m l^{2} C_{1}{ }^{2} C_{2}{ }^{2} \\
& +2 m x l C_{2} S_{1}, m_{12}=-m l S_{2}, m_{13}=m x S_{2}, \\
& m_{14}=-m l^{2} C_{1} C_{2} S_{2}, m_{15}=m l^{2} S_{1}+m x l C_{2}, \\
& m_{21}=-m l S_{2}, m_{22}=M_{t}+m, m_{23}=m C_{2} S_{1} \text {, } \\
& m_{24}=m l C_{1} C_{2}, m_{25}=-m l S_{1} S_{2}, m_{31}=m x S_{2}, \\
& m_{32}=m C_{2} S_{1}, m_{33}=m, m_{34}=m_{35}=0 \text {, } \\
& m_{41}=-m l^{2} C_{1} C_{2} S_{2}, m_{42}=m l C_{1} C_{2}, m_{43}=0 \text {, } \\
& m_{44}=m l^{2} C_{2}{ }^{2}, m_{45}=0, m_{51}=m l^{2} S_{1}+m x l C_{2} \text {, } \\
& m_{52}=-m l S_{1} S_{2}, m_{53}=m_{54}=0, m_{55}=m l^{2} \text {, } \\
& \boldsymbol{C}(\boldsymbol{q}, \dot{\boldsymbol{q}})=\left[\begin{array}{lllll}
c_{11} & c_{12} & c_{13} & c_{14} & c_{15} \\
c_{21} & c_{22} & c_{23} & c_{24} & c_{25} \\
c_{31} & c_{32} & c_{33} & c_{34} & c_{35} \\
c_{41} & c_{42} & c_{43} & c_{44} & c_{45} \\
c_{51} & c_{52} & c_{53} & c_{54} & c_{55}
\end{array}\right], \\
& c_{11}=m x \dot{x}+M_{t} x \dot{x}+m l C_{2} S_{1} \dot{x}+m l \dot{l} \\
& -m l C_{1}{ }^{2} C_{2}{ }^{2} \dot{l}+m x C_{2} S_{1} \dot{l}+m l^{2} C_{1} C_{2}{ }^{2} S_{1} \dot{\theta}_{1} \\
& +m x l C_{1} C_{2} \dot{\theta}_{1}+m l^{2} C_{1}{ }^{2} C_{2} S_{2} \dot{\theta}_{2}-m x l S_{1} S_{2} \dot{\theta}_{2} \text {, } \\
& c_{12}=m x \dot{\alpha}+M_{t} x \dot{\alpha}+m l C_{2} S_{1} \dot{\alpha}, c_{13}=m l \dot{\alpha} \\
& -m l C_{1}{ }^{2} C_{2}{ }^{2} \dot{\alpha}+m x C_{2} S_{1} \dot{\alpha}-m l C_{1} C_{2} S_{2} \dot{\theta}_{1} \\
& +m x C_{2} \dot{\theta}_{2}+m l S_{1} \dot{\theta}_{2}, c_{14}=m l^{2} C_{1} C_{2}{ }^{2} S_{1} \dot{\alpha} \\
& +m x l C_{1} C_{2} \dot{\alpha}-m l C_{1} C_{2} S_{2} \dot{l}+m l^{2} C_{2} S_{1} S_{2} \dot{\theta}_{1} \\
& +m l^{2} C_{1} \dot{\theta}_{2}-m l^{2} C_{1} C_{2}^{2} \dot{\theta}_{2}, \\
& c_{15}=m l^{2} C_{1}{ }^{2} C_{2} S_{2} \dot{\alpha}-m x l S_{1} S_{2} \dot{\alpha}+m x C_{2} \dot{l} \\
& +m l S_{1} \dot{l}+m l^{2} C_{1} \dot{\theta}_{1}-m l^{2} C_{1} C^{2}{ }_{2} \dot{\theta}_{1} \\
& -m x l S_{2} \dot{\theta}_{2}, c_{21}=-m x \dot{\alpha}-M x \dot{\alpha} \\
& -m l C_{2} S_{1} \dot{\alpha}-m S_{2} \dot{l}-m l C_{2} \dot{\theta}_{2}, c_{22}=0, \\
& c_{23}=-m S_{2} \dot{\alpha}+m C_{1} C_{2} \dot{\theta}_{1}-m S_{1} S_{2} \dot{\theta}_{2} \text {, } \\
& c_{24}=m C_{1} C_{2} \dot{l}-m l C_{2} S_{1} \dot{\theta}_{1}-m l C_{1} S_{2} \dot{\theta}_{2} \text {, } \\
& c_{25}=-m l C_{2} \dot{\alpha}-m S_{1} S_{2} \dot{l}-m l C_{1} S_{2} \dot{\theta}_{1} \\
& -m l C_{2} S_{1} \dot{\theta}_{2}, c_{31}=-m l \dot{\alpha}+m l C_{1}{ }^{2} C_{2}{ }^{2} \dot{\alpha}
\end{aligned}
$$

$$
\begin{aligned}
& -m x C_{2} S_{1} \dot{\alpha}+m S_{2} \dot{x}+m l C_{1} C_{2} S_{2} \dot{\theta}_{1}-m l S_{1} \dot{\theta}_{2}, \\
& c_{32}=m S_{2} \dot{\alpha}, c_{33}=0, c_{34}=m l C_{1} C_{2} S_{2} \dot{\alpha} \\
& -m l C_{2}{ }^{2} \dot{\theta}_{1}, c_{35}=-m l S_{1} \dot{\alpha}-m l \dot{\theta}_{2}, \\
& c_{41}=-m l^{2} C_{1} C_{2}{ }^{2} S_{1} \dot{\alpha}-m x l C_{1} C_{2} \dot{\alpha} \\
& -m l C_{1} C_{2} S_{2} \dot{l}-m l^{2} C_{1} C_{2}{ }^{2} \dot{\theta}_{2}, c_{42}=0, \\
& c_{43}=-m l C_{1} C_{2} S_{2} \dot{\alpha}+m l C_{2}^{2} \dot{\theta}_{1}, \\
& c_{44}=m l C_{2}{ }^{2} l-m l^{2} C_{2} S_{2} \dot{\theta}_{2}, \\
& c_{45}=-m l^{2} C_{1} C_{2}{ }^{2} \dot{\alpha}-m l^{2} C_{2} S_{2} \dot{\theta}_{1}, \\
& c_{51}=-m l^{2} C_{1}{ }^{2} C_{2} S_{2} \dot{\alpha}+m l x S_{1} S_{2} \dot{\alpha}+m l C_{2} \dot{x} \\
& +m l S_{1} \dot{i}+m l^{2} C_{1} C_{2}^{2} \dot{\theta}_{1}, c_{52}=m l C_{2} \dot{\alpha}, \\
& c_{53}=m l \dot{\theta}_{2}+m l S_{1} \dot{\alpha}, c_{54}=m l^{2} C_{1} C_{2}^{2} \dot{\alpha} \\
& +m l^{2} C_{2} S_{2} \dot{\theta}_{1}, c_{55}=m l l, \\
& \boldsymbol{G}(\boldsymbol{q})=\left[g_{1} g_{2} g_{3} g_{4} g_{5}\right]^{T}, \\
& g_{1}=0, g_{2}=0, g_{3}=-m g C_{1} C_{2}, g_{4}=m g l C_{2} S_{1}, \\
& g_{5}=m g l C_{1} S_{2},
\end{aligned}
$$

where $m$ and $M_{t}$ denote the payload mass and the trolley mass, respectively. $J_{0}$ is the rotational inertia of the jib and $g$ is the earth acceleration. $\alpha(t), x(t)$, $l(t), \theta_{1}(t)$ and $\theta_{2}(t)$ respectively represent the jib rotational angle, the trolley position, the rope length, the payload swing angle in the jib vertical plane and the payload swing angle out of that plane as shown

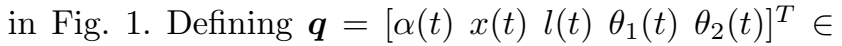
$R^{5}$ is the tower crane system generalized coordinates, $\boldsymbol{M}(\boldsymbol{q}) \in R^{5 \times 5}$ denotes the inertia matrix, $\boldsymbol{C}(\boldsymbol{q}, \dot{\boldsymbol{q}}) \in$ $R^{5 \times 5}$ represents the centripetal-Coriolis matrix, $\boldsymbol{G}(\boldsymbol{q}) \in$ $R^{5}$ is the gravity-related vector, $\boldsymbol{U}=\left[\begin{array}{lllll}T & F_{x} & F_{l} & 0 & 0\end{array}\right]^{T} \in$

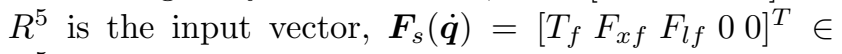
$R^{5}$ denotes the nonlinear friction-related vector, $\boldsymbol{D}=$ $\left[\begin{array}{lllll}d_{1} & d_{2} & d_{3} & d_{4} & d_{5}\end{array}\right]^{T} \in R^{5}$ represents the disturbance vector, including model parameters uncertainties, the unconsidered nonlinear factors and external disturbances, etc. Besides, for ease of reading, we set $S_{i}=\sin \theta_{i}$ and $C_{i}=\cos \theta_{i}(i=1,2)$ in the full paper.

In addition, $T_{f}, F_{x f}$ and $F_{l f}$ are respectively produced by the motion of the jib and trolley, and the rope hoisting/lowing. The following feed-forward friction compensation model is used [31]:

$$
\boldsymbol{F}_{s}(i)=f_{i 1} \tanh \left(\frac{\dot{\boldsymbol{q}}(i)}{\varepsilon}\right)+f_{i 2}|\dot{\boldsymbol{q}}(i)| \dot{\boldsymbol{q}}(i), i=1,2,3,
$$

where $f_{i 1}, f_{i 2}, \varepsilon$ denote the friction-related coefficients, noticing that the precise values of them are difficult to be chosen unless by cumbersome and unpractical repetition test.

The following assumption needs to be declared for ease of analysis:

- The disturbance vector $\boldsymbol{D}$ includes model parameters uncertainties, unconsidered nonlinear factors 


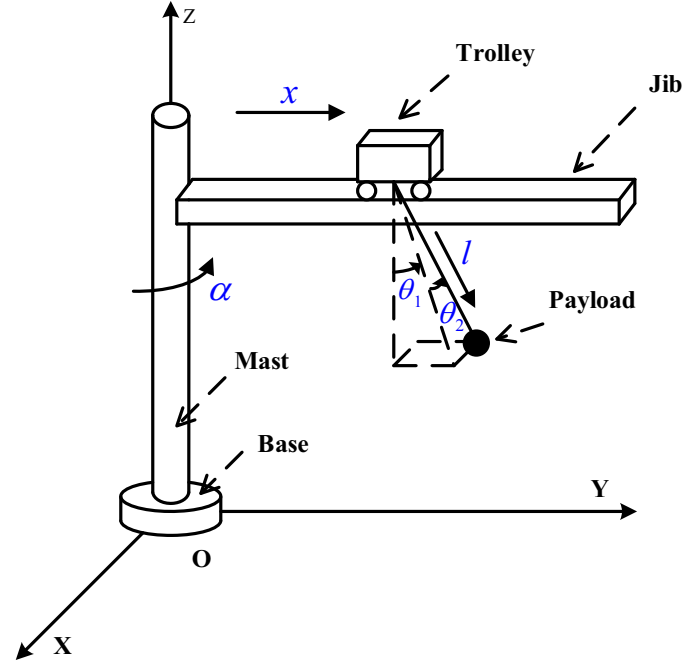

Fig. 1 Tower crane model

and external disturbances, etc., and $\boldsymbol{D}$ is usually assumed to be limited, that is [8] [32],

$\left|d_{i}\right| \leq \nu_{i}, i=1, \ldots, 5$,

where $\nu_{i}$ denotes the upper limit of the aggregate uncertainties of each DOF.

To quickly suppress the payload swing and meanwhile drive the jib, trolley and rope from the initial angle, position and length to the target values are the control objective, which can be presented mathematically as follows:

$\lim _{t \rightarrow \infty} \boldsymbol{q}=\boldsymbol{q}_{d}$

where $\boldsymbol{q}_{d}=\left[\begin{array}{lllll}\alpha_{d} & x_{d} & l_{d} & 0 & 0\end{array}\right]^{T}, \alpha_{d}$ represents the target angle of the jib, and $x_{d}$ and $l_{d}$ are the target position and target length of the trolley and rope, respectively.

To avoid problems, big initial actuators output values and unsmooth running process, the proposed method tracks the reference trajectories to implement positioning, which have the following properties:

1) The final values for velocity and acceleration of jib, trolley and rope should be zero at $t_{d}$,

$$
\begin{array}{r}
\alpha_{r}(t)=\alpha_{d}, \dot{\alpha}_{r}(t)=0, \ddot{\alpha}_{r}(t)=0, t \geq t_{d}, \\
x_{r}(t)=x_{d}, \dot{x}_{r}(t)=0, \ddot{x}_{r}(t)=0, t \geq t_{d}, \\
l_{r}(t)=l_{d}, \dot{l}_{r}(t)=0, \ddot{l}_{r}(t)=0, t \geq t_{d},
\end{array}
$$

where $\alpha_{r}(t), x_{r}(t)$ and $l_{r}(t)$ mean the reference trajectories of the jib, trolley and rope, respectively.

2) For safety reasons and actual situations, the reference trajectories do not have infinite values state, that is,

$$
\begin{aligned}
\alpha_{r}(t), \dot{\alpha}_{r}(t), \ddot{\alpha}_{r}(t) & \in L_{\infty}, \\
x_{r}(t), \dot{x}_{r}(t), \ddot{x}_{r}(t) & \in L_{\infty}, \\
l_{r}(t), \dot{l}_{r}(t), \ddot{l}_{r}(t) & \in L_{\infty} .
\end{aligned}
$$

3) The definition of initial values for each target trajectories are as follows:

$$
\begin{array}{r}
\alpha_{r}(0)=\alpha_{0}, \dot{\alpha}_{r}(0)=0, \ddot{\alpha}_{r}(0)=0, \\
x_{r}(0)=x_{0}, \dot{x}_{r}(0)=0, \ddot{x}_{r}(0)=0, \\
l_{r}(0)=l_{0}, \dot{l}_{r}(0)=0, \ddot{l}_{r}(0)=0,
\end{array}
$$

where $\alpha_{0}, x_{0}$ and $l_{0}$ denote the initial angle, initial position and initial length of the jib, trolley and rope, respectively.

After the above analysis, the following reference trajectories that satisfy the requirements are utilized:

$$
r_{i}=\left\{\begin{array}{c}
\left(\boldsymbol{q}(i)_{d}-\boldsymbol{q}(i)_{0}\right)\left(\frac{t}{t_{d}}-\frac{\sin \left(\frac{2 \pi t}{t_{d}}\right)}{2 \pi}\right) \\
\quad+\boldsymbol{q}(i)_{0}, t \in\left[0, t_{d}\right) \\
\boldsymbol{q}(i)_{d}, t \in\left[t_{d},+\infty\right]
\end{array},\right.
$$

where $i=1,2,3, r_{1}=\alpha_{r}(t), r_{2}=x_{r}(t), r_{3}=l_{r}(t)$, $\boldsymbol{q}(1)_{d}=\alpha_{d}, \boldsymbol{q}(2)_{d}=x_{d}, \boldsymbol{q}(3)_{d}=l_{d} \leq l_{u}, \boldsymbol{q}(1)_{0}=\alpha_{0}$, $\boldsymbol{q}(2)_{0}=x_{0}, \boldsymbol{q}(3)_{0}=l_{0} \geq l_{l}$, and $l_{l} \leq l \leq l_{u}, l_{l}$ is a positive value represented the lower limiting value of the rope length, and $l_{u}$ denotes the upper limit value. We define $\boldsymbol{q}_{r}(t)=\left[\begin{array}{lll}\alpha_{r}(t) x_{r}(t) l_{r}(t) & 0 & 0\end{array}\right]^{T}$.

Remark 1: The initial values setting of all reference trajectories is essential in actual tower cranes operation circumstances. Firstly, the position of the trolley and the length of the rope can not be zero considering tower cranes physical structure and safety. On the other hand, the values of the jib angle, the trolley position and the rope length are always changed in continuous positioning tasks, thus to set reference trajectories initial values is more practical.

\section{Main Results}

\subsection{Controller Design}

After simple mathematic operations, the Eq. (1) changes its form to the following result:

$\ddot{\boldsymbol{q}}=\boldsymbol{M}(\boldsymbol{q})^{-1}\left(\boldsymbol{U}-\boldsymbol{F}_{s}(\dot{\boldsymbol{q}})+\boldsymbol{D}-\boldsymbol{C}(\boldsymbol{q}, \dot{\boldsymbol{q}}) \dot{\boldsymbol{q}}-\boldsymbol{G}(\boldsymbol{q})\right)$,

and Eq. (9) extracts $\ddot{\boldsymbol{q}}$ from dynamic equation Eq. (1). Then, the item of $\boldsymbol{D}$ is separated, and we have

$$
\begin{aligned}
\ddot{\boldsymbol{q}}= & \boldsymbol{M}(\boldsymbol{q})^{-1}\left(\boldsymbol{U}-\boldsymbol{F}_{s}(\dot{\boldsymbol{q}})-\boldsymbol{C}(\boldsymbol{q}, \dot{\boldsymbol{q}}) \dot{\boldsymbol{q}}\right. \\
& \left.-\boldsymbol{G}_{c}^{\prime}(\boldsymbol{q})-\boldsymbol{G}^{\prime}(\boldsymbol{q})\right)+\boldsymbol{M}(\boldsymbol{q})^{-1} \boldsymbol{D},
\end{aligned}
$$

where $\boldsymbol{G}_{c}^{\prime}=\left[\begin{array}{lllll}0 & 0 & -m g C_{1} C_{2} & 0 & 0\end{array}\right]^{T} \in R^{5}, \boldsymbol{G}^{\prime}(\boldsymbol{q})=\left[\begin{array}{lll}0 & 0 & 0\end{array}\right.$ $\left.m g l C_{2} S_{1} m g l C_{1} S_{2}\right]^{T} \in R^{5}$.

Taking out the driving force/torque and composing a new input vector $\boldsymbol{U}_{a}=\left[\begin{array}{lll}T & F_{x} & F_{l}\end{array}\right]^{T} \in R^{3}$ to analysis, the following equation is derived:

$$
\begin{array}{r}
\ddot{\boldsymbol{q}}=\boldsymbol{\Gamma}(\boldsymbol{q}, \dot{\boldsymbol{q}})+\boldsymbol{\Phi}(\boldsymbol{q})\left(\boldsymbol{U}_{a}-\boldsymbol{F}_{s a}(\dot{\boldsymbol{q}})-\boldsymbol{G}_{c}(\boldsymbol{q})\right) \\
+\boldsymbol{M}(\boldsymbol{q})^{-1} \boldsymbol{D},
\end{array}
$$


where $\boldsymbol{F}_{s a}(\dot{\boldsymbol{q}})=\left[\begin{array}{lll}T_{f} & F_{x f} & F_{l f}\end{array}\right]^{T} \in R^{3}, \boldsymbol{G}_{c}=\left[\begin{array}{lll}0 & 0 & -\end{array}\right.$ $\left.m g C_{1} C_{2}\right]^{T} \in R^{3}$ and the detailed expression of $\boldsymbol{\Gamma}(\boldsymbol{q}, \dot{\boldsymbol{q}}) \in$ $R^{5}$ and $\boldsymbol{\Phi}(\boldsymbol{q}) \in R^{5 \times 3}$ are as follows:

$\boldsymbol{\Gamma}(\boldsymbol{q}, \dot{\boldsymbol{q}})=\left[\begin{array}{lllll}a_{1} & a_{2} & a_{3} & a_{4} & a_{5}\end{array}\right]^{T}$,

$a_{1}=\frac{-2 M_{t} \dot{\alpha} \dot{x} x}{M_{t} x^{2}+J_{0}}, a_{2}=x \dot{\alpha}^{2}$,

$a_{3}=\frac{\left(\begin{array}{l}M_{t} \dot{\theta}_{2}^{2} l x^{2}+M_{t} \dot{\alpha}^{2} l x^{2}+J_{0} \dot{\theta}_{2}^{2} l m \\ +J_{0} \dot{\alpha}^{2} l+M_{t} \dot{\theta}_{1}^{2} l x^{2} C_{2}{ }^{2}+J_{0} \dot{\theta}_{1}^{2} l C_{2}{ }^{2} \\ -2 J_{0} \dot{\alpha} \dot{x} S_{2}-2 M_{t} \dot{\theta}_{1} \dot{\alpha} l x^{2} C_{1} C_{2} S_{2} \\ +2 M_{t} \dot{\theta}_{2} \dot{\alpha} l x^{2} S_{1}+2 J_{0} \dot{\theta}_{2} \dot{\alpha} l S_{1} \\ -M_{t} \dot{\alpha}^{2} l x^{2} C_{1}^{2} C_{2}^{2}-J_{0} \dot{\alpha}^{2} l C_{1}^{2} C_{2}^{2} \\ -2 J_{0} \dot{\theta}_{1} \dot{\alpha} l C_{1} C_{2} S_{2}\end{array}\right)}{M_{t} x^{2}+J_{0}}$,

$a_{4}=\frac{\left(\begin{array}{l}-M_{t} g x^{2} S_{1}-J_{0} g S_{1}-2 M_{t} \dot{\theta}_{1} i x^{2} C_{2} \\ -2 J_{0} \dot{\theta}_{1} l C_{2}+2 M_{t} \dot{\alpha} \dot{l} x^{2} C_{1} S_{2} \\ +2 J_{0} \dot{\dot{\alpha}} i C_{1} S_{2}+2 M_{t} \dot{\theta}_{1} \dot{\theta}_{2} l x^{2} S_{2} \\ +2 J_{0} \dot{\theta}_{1} \dot{\theta}_{2} l S_{2}-2 M_{t} \dot{\alpha} \dot{x} l x C_{1} S_{2} \\ +M_{t} \dot{\alpha}^{2} l x^{2} C_{1} C_{2} S_{1}+J_{0} \dot{\alpha}^{2} l C_{1} C_{2} S_{1} \\ +2 M_{t} \dot{\theta}_{2} \dot{\alpha} l x^{2} C_{1} C_{2}+2 J_{0} \dot{\theta}_{2} \dot{\alpha} l C_{1} C_{2}\end{array}\right)}{l C_{2}\left(M_{t} x^{2}+J_{0}\right)}$,

$a_{5}=\frac{\left(\begin{array}{l}-2 M_{t} \dot{\theta}_{2} i x^{2}-2 M_{t} \dot{\theta}_{1} \dot{\alpha} l x^{2} C_{1} C_{2}{ }^{2} \\ -2 J_{0} \dot{\alpha} \dot{x} C_{2}-M_{t} \dot{\theta}_{1}^{2} l x^{2} S_{2} C_{2} \\ -J_{0} \dot{\theta}_{1}^{2} l S_{2} C_{2}-2 M_{t} \dot{\alpha} \dot{l} x^{2} S_{1} \\ -J_{0} g C_{1} S_{2}-2 J_{0} \dot{\theta}_{1} \dot{\alpha} l C_{1} C_{2}{ }^{2} \\ -2 J_{0} \dot{\theta}_{2} \dot{l}+M_{t} \dot{\alpha}^{2} l x^{2} C_{1}{ }^{2} C_{2} S_{2} \\ -M_{t} g x^{2} C_{1} S_{2}+2 M_{t} \dot{\alpha} \dot{x} l x S_{1} \\ -2 J_{0} \dot{\alpha} i S_{1}+J_{0} \dot{\alpha}^{2} l C_{1}{ }^{2} C_{2} S_{2}\end{array}\right)}{l\left(M_{t} x^{2}+J_{0}\right)}$,

$\boldsymbol{\Phi}(\boldsymbol{q})=\left[\begin{array}{lllll}b_{11} & b_{21} & b_{31} & b_{41} & b_{51} \\ b_{12} & b_{22} & b_{32} & b_{42} & b_{52} \\ b_{13} & b_{23} & b_{33} & b_{43} & b_{53}\end{array}\right]^{T}$,

$b_{11}=\frac{1}{M_{t} x^{2}+J_{0}}, b_{12}=0, b_{13}=\frac{-x S_{2}}{M_{t} x^{2}+J_{0}}$,

$b_{21}=0, b_{22}=\frac{1}{M_{t}}, b_{23}=\frac{-C_{2} S_{1}}{M_{t}}$,

$b_{31}=\frac{-x S_{2}}{M_{t} x^{2}+J_{0}}, b_{32}=\frac{-S_{1} C_{2}}{M_{t}}$,

$b_{33}=\frac{\left(\begin{array}{l}M_{t}^{2} x^{2}+J_{0} M_{t}+M_{t} m x^{2}+J_{0} m C_{2}{ }^{2} \\ -J_{0} m C_{1}^{2} C_{2}^{2}-M_{t} m x^{2} C_{1}{ }^{2} C_{2}{ }^{2}\end{array}\right)}{M_{t} m\left(M_{t} x^{2}+J_{0}\right)}$,

$b_{41}=\frac{C_{1} S_{2}}{C_{2}\left(M_{t} x^{2}+J_{0}\right)}, b_{42}=\frac{-C_{1}}{M_{t} l C_{2}}$,

$b_{43}=\frac{C_{1}\left(\begin{array}{l}-M_{t} l x+J_{0} C_{2} S_{1} \\ +M_{t} l x C_{2}{ }^{2}+M_{t} x^{2} C_{2} S_{1}\end{array}\right)}{M_{t} l C_{2}\left(M_{t} x^{2}+J_{0}\right)}$,

$b_{51}=\frac{-x C_{2}-l S_{1}}{l\left(M_{t} x^{2}+J_{0}\right)}, b_{52}=\frac{S_{1} S_{2}}{M_{t} l}$,
$b_{53}=\frac{S_{2}\left(\begin{array}{l}-J_{0} C_{2}+J_{0} C_{1}{ }^{2} C_{2} \\ +M_{t} x^{2} C_{1}^{2} C_{2}+M_{t} l x S_{1}\end{array}\right)}{M_{t} l\left(M_{t} x^{2}+J_{0}\right)}$.

To achieve smooth and fast convergence effect, the following adaptive sliding mode surface is constructed,

$$
\begin{aligned}
& \boldsymbol{S}=\dot{\boldsymbol{e}}+\boldsymbol{\Lambda} \hat{\boldsymbol{\Delta}}(t) \boldsymbol{e}=\left[\begin{array}{c}
\dot{\alpha}-\dot{\alpha}_{r}(t) \\
\dot{x}-\dot{x}_{r}(t) \\
\dot{i}-\dot{l}_{r}(t) \\
\dot{\theta}_{1} \\
\dot{\theta}_{2}
\end{array}\right]+\left[\begin{array}{ccccc}
k_{1} & 0 & 0 & 0 & 0 \\
0 & k_{2} & 0 & 0 & 0 \\
0 & 0 & k_{3} & 0 & 0 \\
0 & 0 & 0 & k_{4} & 0 \\
0 & 0 & 0 & 0 & k_{5}
\end{array}\right] \\
& {\left[\begin{array}{ccccc}
\hat{\lambda}_{1}(t) & 0 & 0 & 0 & 0 \\
0 & \hat{\lambda}_{2}(t) & 0 & 0 & 0 \\
0 & 0 & \hat{\lambda}_{3}(t) & 0 & 0 \\
0 & 0 & 0 & \hat{\lambda}_{4}(t) & 0 \\
0 & 0 & 0 & 0 & \hat{\lambda}_{5}(t)
\end{array}\right]\left[\begin{array}{c}
\alpha-\alpha_{r}(t) \\
x-x_{r}(t) \\
l-l_{r}(t) \\
\theta_{1} \\
\theta_{2}
\end{array}\right]}
\end{aligned}
$$

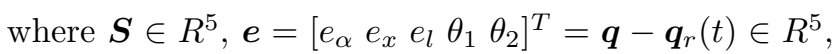
$\boldsymbol{\Lambda}=\operatorname{diag}\left\{k_{1}, \ldots, k_{5}\right\} \in R^{5 \times 5}$ where its diagonal elements are all positive, $\hat{\boldsymbol{\Delta}}(t)=\operatorname{diag}\left\{\hat{\lambda}_{1}(t), \ldots, \hat{\lambda}_{5}(t)\right\} \in$ $R^{5 \times 5}$ is the adaptive diagonal matrix where its diagonal elements is time-varying by system condition. Noting that the sliding surface $\boldsymbol{S}$ has the following properties: 1 ) it is a vector in five dimensions; 2 ) it has a constant gain and a adaptive gain diagonal matrix; 3 ) it contain$\mathrm{s}$ reference trajectory. In a word, the sliding surface is adaptive and contains trajectory tracking demand of all DOF.

The following sliding mode reaching law is selected,

$$
\begin{aligned}
\dot{\boldsymbol{S}}= & -\mathbf{I} \boldsymbol{S}-\boldsymbol{H} \operatorname{sgn}(\boldsymbol{S}) \\
= & -\left[\begin{array}{l}
\mu_{1}\left(\dot{e}_{\alpha}+k_{1} \hat{\lambda}_{1}(t) e_{\alpha}\right) \\
\mu_{2}\left(\dot{e}_{x}+k_{2} \hat{\lambda}_{2}(t) e_{x}\right) \\
\mu_{3}\left(\dot{e}_{l}+k_{3} \hat{\lambda}_{3}(t) e_{l}\right) \\
\mu_{4}\left(\dot{\theta}_{1}+k_{4} \hat{\lambda}_{4}(t) \theta_{1}\right) \\
\mu_{5}\left(\dot{\theta}_{2}+k_{5} \hat{\lambda}_{5}(t) \theta_{2}\right)
\end{array}\right] \\
& -\left[\begin{array}{l}
\gamma_{1} \operatorname{sgn}\left(\dot{e}_{\alpha}+k_{1} \hat{\lambda}_{1}(t) e_{\alpha}\right) \\
\gamma_{2} \operatorname{sgn}\left(\dot{e}_{x}+k_{2} \hat{\lambda}_{2}(t) e_{x}\right) \\
\gamma_{3} \operatorname{sgn}\left(\dot{e}_{l}+k_{3} \hat{\lambda}_{3}(t) e_{l}\right) \\
\gamma_{4} \operatorname{sgn}\left(\dot{\theta}_{1}+k_{4} \hat{\lambda}_{4}(t) \theta_{1}\right) \\
\gamma_{5} \operatorname{sgn}\left(\dot{\theta}_{2}+k_{5} \hat{\lambda}_{5}(t) \theta_{2}\right)
\end{array}\right],
\end{aligned}
$$

where $\mathbf{I}=\operatorname{diag}\left\{\mu_{1}, \ldots, \mu_{5}\right\} \in R^{5 \times 5}$ in which its diagonal elements are all positive numbers to be designed, $\mathbf{H}=\operatorname{diag}\left\{\gamma_{1}, \ldots, \gamma_{5}\right\} \in R^{5 \times 5}$ where its diagonal elements are also positive and to be determined. Therefore, the sliding mode converging condition is that

$$
\begin{aligned}
\boldsymbol{S}^{T} \dot{\boldsymbol{S}} & =\boldsymbol{S}^{T}(-\mathbf{I} \boldsymbol{S}-\mathbf{H} \operatorname{sgn}(\boldsymbol{S})) \\
& =-\boldsymbol{S}^{T} \mathbf{I} \boldsymbol{S}-\boldsymbol{S}^{T} \mathbf{H} \operatorname{sgn}(\boldsymbol{S}) \leq 0 .
\end{aligned}
$$


Taking the derivative of $\boldsymbol{S}$ with respect to time, and substituting (11) into it, the result is that

$$
\begin{aligned}
\dot{\boldsymbol{S}}= & \ddot{\boldsymbol{e}}+\boldsymbol{\Lambda} \dot{\hat{\boldsymbol{\Delta}}}(t) \boldsymbol{e}+\boldsymbol{\Lambda} \hat{\boldsymbol{\Delta}}(t) \dot{\boldsymbol{e}} \\
= & \ddot{\boldsymbol{q}}-\ddot{\boldsymbol{q}}_{r}(t)+\boldsymbol{\Lambda} \dot{\hat{\boldsymbol{\Delta}}}(t) \boldsymbol{e}+\boldsymbol{\Lambda} \hat{\boldsymbol{\Delta}}(t) \dot{\boldsymbol{e}} \\
= & \boldsymbol{\Gamma}(\boldsymbol{q}, \dot{\boldsymbol{q}})+\boldsymbol{\Phi}(\boldsymbol{q})\left(\boldsymbol{U}_{a}-\boldsymbol{F}_{s a}(\dot{\boldsymbol{q}})-\boldsymbol{G}_{c}(\boldsymbol{q})\right) \\
& +\boldsymbol{M}(\boldsymbol{q})^{-1} \boldsymbol{D}-\ddot{\boldsymbol{q}}_{r}(t)+\boldsymbol{\Lambda} \dot{\hat{\boldsymbol{\Delta}}}(t) \boldsymbol{e}+\boldsymbol{\Lambda} \hat{\boldsymbol{\Delta}}(t) \dot{\boldsymbol{e}}
\end{aligned}
$$

The input vector $U_{a}$ is composed of two parts, and it is that

$$
\begin{aligned}
\boldsymbol{U}_{a}= & \boldsymbol{U}_{e q}+\boldsymbol{U}_{s w}, \\
\boldsymbol{U}_{e q}= & \left(\boldsymbol{\Phi}(\boldsymbol{q})^{T} \boldsymbol{\Phi}(\boldsymbol{q})\right)^{-1} \boldsymbol{\Phi}(\boldsymbol{q})^{T}\left(-\boldsymbol{\Gamma}(\boldsymbol{q}, \dot{\boldsymbol{q}})-\boldsymbol{M}^{-1} \boldsymbol{D}\right. \\
& \left.+\ddot{\boldsymbol{q}}_{r}(t)-\boldsymbol{\Lambda} \dot{\hat{\boldsymbol{\Delta}}}(t) \boldsymbol{e}-\boldsymbol{\Lambda} \hat{\boldsymbol{\Delta}}(t) \dot{\boldsymbol{e}}\right)+\boldsymbol{F}_{s a}+G_{c}(\boldsymbol{q}),
\end{aligned}
$$

$\boldsymbol{U}_{s w}=\left(\boldsymbol{\Phi}(\boldsymbol{q})^{T} \boldsymbol{\Phi}(\boldsymbol{q})\right)^{-1} \boldsymbol{\Phi}(\boldsymbol{q})^{T}(-\mathbf{I} \boldsymbol{S}-\mathbf{H} \operatorname{sgn}(\boldsymbol{S}))$.

Remark 2: It can be seen that $\boldsymbol{U}_{e q}$ is designed to make the control system be more robust in external disturbances and parametric uncertainties. $\boldsymbol{U}_{\text {sw }}$ is to make sure that in any case, the system state far from or close to the sliding mode surface, the fast convergence is feasible.

However, the frictional parameters and aggregate disturbances are unmeasured, which means that the controller can not contain these unknown parameters. Thus, we have to design adaptive terms to estimate them. Firstly, the estimated frictional model is presented as follows:

$\hat{\boldsymbol{F}}_{s}(i)=\hat{f}_{i 1} \tanh \left(\frac{\dot{\boldsymbol{q}}(i)}{\varepsilon}\right)+\hat{f}_{i 2}|\dot{\boldsymbol{q}}(i)| \dot{\boldsymbol{q}}(i), i=1,2,3$,

and to facilitate design, the following form is presented:

$$
\hat{\boldsymbol{F}}_{s a}=\boldsymbol{v}_{1}(\dot{\boldsymbol{q}}) \hat{\boldsymbol{\Upsilon}}_{1}+\boldsymbol{v}_{2}(\dot{\boldsymbol{q}}) \hat{\boldsymbol{\Upsilon}}_{2},
$$

with $\hat{\boldsymbol{\Upsilon}}_{1}=\left[\begin{array}{lll}\hat{f}_{11} & \hat{f}_{21} & \hat{f}_{31}\end{array}\right]^{T}, \hat{\boldsymbol{\Upsilon}}_{2}=\left[\begin{array}{lll}\hat{f}_{12} & \hat{f}_{22} & \hat{f}_{32}\end{array}\right]^{T}$,

$$
\hat{f}_{i j}\left(t_{0}\right)=\bar{f}_{i j}>0 \in R^{+}, i=1,2,3, j=1,2,
$$

where $\bar{f}_{i j}$ is a positive constant representing the initial estimated value. $\boldsymbol{v}_{1}(\dot{\boldsymbol{q}})=\operatorname{diag}\left\{\tanh \left(\frac{\dot{\alpha}}{\varepsilon}\right), \tanh \left(\frac{\dot{x}}{\varepsilon}\right)\right.$, $\left.\tanh \left(\frac{i}{\varepsilon}\right)\right\}, \boldsymbol{v}_{2}(\dot{\boldsymbol{q}})=\operatorname{diag}\{|\dot{\alpha}| \dot{\alpha},|\dot{x}| \dot{x},|\dot{l}| \dot{l}\}$. It means the adaptive term $\hat{f}_{i j}$ will update from a set positive value to some value, and $\bar{f}_{i j}$ can be approximately chosen close to real friction parameters through experience.

Hence, $\boldsymbol{U}_{e q}$ and $\boldsymbol{U}_{s w}$ are reconstructed as follows:

$$
\begin{aligned}
\boldsymbol{U}_{e q}= & \left(\boldsymbol{\Phi}(\boldsymbol{q})^{T} \boldsymbol{\Phi}(\boldsymbol{q})\right)^{-1} \boldsymbol{\Phi}(\boldsymbol{q})^{T}\left(-\boldsymbol{\Gamma}(\boldsymbol{q}, \dot{\boldsymbol{q}})-\boldsymbol{M}^{-1} \hat{\boldsymbol{D}}\right. \\
& \left.+\ddot{\boldsymbol{q}}_{r}(t)-\boldsymbol{\Lambda} \dot{\hat{\boldsymbol{\Delta}}}(t) \boldsymbol{e}-\boldsymbol{\Lambda} \hat{\boldsymbol{\Delta}}(t) \dot{\boldsymbol{e}}\right) \\
& +\boldsymbol{v}_{1}(\dot{\boldsymbol{q}}) \hat{\boldsymbol{\Upsilon}}_{1}+\boldsymbol{v}_{2}(\dot{\boldsymbol{q}}) \hat{\boldsymbol{\Upsilon}}_{2}+\hat{\boldsymbol{G}}_{c}(\boldsymbol{q}), \\
\boldsymbol{U}_{s w}= & \left(\boldsymbol{\Phi}(\boldsymbol{q})^{T} \boldsymbol{\Phi}(\boldsymbol{q})\right)^{-1} \boldsymbol{\Phi}(\boldsymbol{q})^{T}(-\mathbf{I} \boldsymbol{S}-\mathbf{H} \operatorname{sgn}(\boldsymbol{S})),
\end{aligned}
$$

where $\hat{\boldsymbol{G}}_{c}(\boldsymbol{q})=\left[\begin{array}{lll}0 & 0 & -\hat{m} g C_{1} C_{2}\end{array}\right]^{T} \in R^{3}, \hat{m}$ represents the estimated value of payload mass with $\hat{m}\left(t_{0}\right)=\bar{m}>$ $0 \in R^{+}, \bar{m}$ denotes the initial estimated value.
In (14), we present an adaptive variable gain matrix $\hat{\boldsymbol{\Delta}}(t)$, and the change law of its diagonal elements are designed as follows:

$\dot{\hat{\lambda}}_{i}(t)=\frac{\boldsymbol{e}(i)\left(\ddot{r}_{i}(t)-a_{i}-k_{i} \hat{\lambda}_{i}(t) \dot{\boldsymbol{e}}(i)\right)}{\left(|\boldsymbol{e}(i)|^{2}+\varepsilon\right) k_{i}}, i=1, \ldots, 5$,

where $\varepsilon$ is a pimping positive constant to avoid zeros in the denominator. This is a adaptive term related to $\boldsymbol{e}$ in the sliding surface vector $\boldsymbol{S}$. On one aspect, $\hat{\lambda}_{i}(t)$ is the gain of $\boldsymbol{e}(i), i=1, \ldots, 5$, which affects the convergence time of error, on the other aspect, the adaptive law of $\hat{\lambda}_{i}(t)$ contains $\boldsymbol{e}(i)$ and $\dot{\boldsymbol{e}}(i)$, which means its value is always adaptively changed until $\boldsymbol{e}(i)=0$ and $\dot{\boldsymbol{e}}(i)=0$. This characteristic reflects the adaptive gain $\hat{\lambda}_{i}(t)$ is sensitive to error and can improve error convergence effect to some extent.

Reformatting the proposed controller and adding payload gravity compensation, we have

$$
\begin{array}{r}
\boldsymbol{U}_{a}=\left(\boldsymbol{\Phi}(\boldsymbol{q})^{T} \boldsymbol{\Phi}(\boldsymbol{q})\right)^{-1} \boldsymbol{\Phi}(\boldsymbol{q})^{T}(-\mathbf{I} \boldsymbol{S}-\mathbf{H} \operatorname{sgn}(\boldsymbol{S}) \\
\left.-\boldsymbol{M}^{-1} \hat{\boldsymbol{D}}\right)+\boldsymbol{v}_{1}(\dot{\boldsymbol{q}}) \hat{\boldsymbol{\Upsilon}}_{1}+\boldsymbol{v}_{2}(\dot{\boldsymbol{q}}) \hat{\boldsymbol{\Upsilon}}_{2}+\hat{\boldsymbol{G}}_{c}(\boldsymbol{q}),
\end{array}
$$

To minimize the chattering effect of sliding mode control, the signum function is replaced by the hyperbolic tangent function, that is,

$$
\begin{array}{r}
\boldsymbol{U}_{a}=\left(\boldsymbol{\Phi}(\boldsymbol{q})^{T} \boldsymbol{\Phi}(\boldsymbol{q})\right)^{-1} \boldsymbol{\Phi}(\boldsymbol{q})^{T}(-\mathbf{I} \boldsymbol{S}-\mathbf{H} \tanh (\boldsymbol{S}) \\
\left.-\boldsymbol{M}^{-1} \hat{\boldsymbol{D}}\right)+\boldsymbol{v}_{1}(\dot{\boldsymbol{q}}) \hat{\boldsymbol{\Upsilon}}_{1}+\boldsymbol{v}_{2}(\dot{\boldsymbol{q}}) \hat{\boldsymbol{\Upsilon}}_{2}+\hat{m} \boldsymbol{G}_{c}^{*}(\boldsymbol{q}),
\end{array}
$$

where $\boldsymbol{G}_{c}^{*}(\boldsymbol{q})=\left[\begin{array}{lll}0 & 0 & -C_{1} C_{2} g\end{array}\right]$ and the adaptive terms update laws are as follows:

$$
\begin{aligned}
& \dot{\hat{\boldsymbol{\Upsilon}}}_{1}=-\boldsymbol{\Pi}_{1}^{-1} \boldsymbol{v}_{1}(\dot{\boldsymbol{q}})^{T} \boldsymbol{\Phi}(\boldsymbol{q})^{T} \boldsymbol{S}, \\
& \dot{\hat{\boldsymbol{\Upsilon}}}_{2}=-\boldsymbol{\Pi}_{2}^{-1} \boldsymbol{v}_{2}(\dot{\boldsymbol{q}})^{T} \boldsymbol{\Phi}(\boldsymbol{q})^{T} \boldsymbol{S}, \\
& \dot{\hat{\boldsymbol{D}}}=\boldsymbol{\Pi}_{d}^{-1}\left(\boldsymbol{M}(\boldsymbol{q})^{-1}\right)^{T} \boldsymbol{S} . \\
& \dot{\hat{m}}=-\frac{1}{\varphi_{m}} \boldsymbol{S}^{T} \boldsymbol{\Phi}(\boldsymbol{q}) \boldsymbol{G}_{c}^{*}(\boldsymbol{q})
\end{aligned}
$$

where $\boldsymbol{\Pi}_{d}=\operatorname{diag}\left\{\varphi_{d 1}, \ldots, \varphi_{d 5}\right\} \in R^{5 \times 5}, \boldsymbol{\Pi}_{1}=\operatorname{diag}$ $\left\{\varphi_{11}, \varphi_{12}, \varphi_{13}\right\} \in R^{3 \times 3}$ and $\boldsymbol{\Pi}_{2}=\operatorname{diag}\left\{\varphi_{21}, \varphi_{22}, \varphi_{23}\right\}$ $\in R^{3 \times 3}$ are all positive diagonal matrices, and, $\varphi_{m}$ is a positive gain.

Remark 3: Many previous researches about adaptive control for cranes (e.g., [9] [11] [12]) only used adaptive technique to estimate frictional parameters or unknown model parameters, where not only the structure of the adaptive part is complicated, but also the external disturbances and the unmodeled non-linearity factors are not considered in theory.

\subsection{Stability Analysis}

Theorem 1 The proposed method is effective in the positioning of the actuated part (i.e., $\alpha, x$ and $l$ ), and 
meanwhile capable of payload swing angles (i.e., $\theta_{1}$ and $\left.\theta_{2}\right)$ suppression. Thus, its mathematical expression is as follows:

$\lim _{t \rightarrow \infty}\left[\alpha x l \theta_{1} \theta_{2} \dot{\alpha} \dot{x} \dot{l} \dot{\theta}_{1} \dot{\theta}_{2} \ddot{\alpha} \ddot{x} \ddot{l} \ddot{\theta}_{1} \ddot{\theta}_{2}\right]^{T}$

$$
=\left[\begin{array}{lllllllllllll}
\alpha_{d} x_{d} l_{d} & 0 & 0 & 0 & 0 & 0 & 0 & 0 & 0 & 0 & 0 & 0 & 0
\end{array}\right]^{T} \text {. }
$$

Proof: The following positive definite function $V$ is designed:

$$
\begin{aligned}
V=\frac{1}{2} \boldsymbol{S}^{T} \boldsymbol{S}+ & \frac{1}{2} \sum_{i=1}^{5} \varphi_{d i} \tilde{d}_{i}^{2}+\frac{1}{2} \sum_{i=1}^{3} \varphi_{i 1} \tilde{f}_{i 1}^{2} \\
& +\frac{1}{2} \sum_{i=1}^{3} \varphi_{i 2} \tilde{f}_{i 2}^{2}+\frac{1}{2} \varphi_{m} \tilde{m}^{2},
\end{aligned}
$$

where $\tilde{d}_{i}=d_{i}-\hat{d}_{i}, \tilde{f}_{i 1}=f_{i 1}-\hat{f}_{i 1}, \tilde{f}_{i 2}=f_{i 2}-\hat{f}_{i 2}$ and $\tilde{m}=m-\hat{m}$ denote the estimated error of unknown parameters.

Then, by taking the time derivative of (33) and considering (17), (22), (25) and (26), we have

$$
\begin{aligned}
& \dot{V}=\boldsymbol{S}^{T} \dot{\boldsymbol{S}}-\sum_{i=1}^{3} \varphi_{i 1} \tilde{f}_{i 1} \dot{\hat{f}}_{i 1}-\sum_{i=1}^{3} \varphi_{i 2} \tilde{f}_{i 2} \dot{\hat{f}}_{i 2} \\
& -\sum_{i=1}^{5} \varphi_{d i} \tilde{d}_{i} \dot{\hat{d}}_{i}-\varphi_{m} \tilde{m} \dot{\hat{m}} \\
& =\boldsymbol{S}^{T} \dot{\boldsymbol{S}}-\tilde{\boldsymbol{D}}^{T} \boldsymbol{\Pi}_{d} \dot{\hat{\boldsymbol{D}}}-\tilde{\boldsymbol{\Upsilon}}_{1}^{T} \boldsymbol{\Pi}_{1} \dot{\hat{\boldsymbol{\Upsilon}}}_{1} \\
& -\tilde{\boldsymbol{\Upsilon}}_{2}^{T} \boldsymbol{\Pi}_{2} \dot{\hat{\boldsymbol{\Upsilon}}}_{2}-\varphi_{m} \tilde{m} \dot{\hat{m}} \\
& =\boldsymbol{S}^{T}\left(\boldsymbol{\Gamma}(\boldsymbol{q}, \dot{\boldsymbol{q}})+\boldsymbol{\Phi}(\boldsymbol{q})\left(\boldsymbol{U}_{a}-\boldsymbol{F}_{s a}-\boldsymbol{G}_{c}(\boldsymbol{q})\right)\right. \\
& \left.+\boldsymbol{M}^{-1} \boldsymbol{D}-\ddot{\boldsymbol{q}}_{r}(t)+\boldsymbol{\Lambda} \dot{\hat{\boldsymbol{\Delta}}}(t) \boldsymbol{e}+\boldsymbol{\Lambda} \hat{\boldsymbol{\Delta}}(t) \dot{\boldsymbol{e}}\right) \\
& -\tilde{\boldsymbol{D}}^{T} \boldsymbol{\Pi}_{d} \dot{\hat{\boldsymbol{D}}}-\tilde{\boldsymbol{\Upsilon}}_{1}^{T} \boldsymbol{\Pi}_{1} \dot{\hat{\boldsymbol{\Upsilon}}}_{1}-\tilde{\boldsymbol{\Upsilon}}_{2}^{T} \boldsymbol{\Pi}_{2} \dot{\hat{\boldsymbol{\Upsilon}}}_{2}-\varphi_{m} \tilde{m} \dot{\hat{m}} \\
& =\boldsymbol{S}^{T}\left(-\mathbf{I} \boldsymbol{S}-\mathbf{H} \operatorname{sgn}(\boldsymbol{S})-\boldsymbol{M}^{-1} \hat{\boldsymbol{D}}\right. \\
& +\boldsymbol{\Phi}(\boldsymbol{q})\left(\boldsymbol{v}_{1}(\dot{\boldsymbol{q}}) \hat{\boldsymbol{\Upsilon}}_{1}+\boldsymbol{v}_{2}(\dot{\boldsymbol{q}}) \hat{\boldsymbol{\Upsilon}}_{2}-\boldsymbol{F}_{s a}+\hat{m} \boldsymbol{G}_{c}^{*}(\boldsymbol{q})\right. \\
& \left.\left.-\boldsymbol{G}_{c}(\boldsymbol{q})\right)+\boldsymbol{M}^{-1} \boldsymbol{D}\right)-\tilde{\boldsymbol{D}}^{T} \boldsymbol{\Pi}_{d} \dot{\hat{\boldsymbol{D}}} \\
& -\tilde{\boldsymbol{\Upsilon}}_{1}^{T} \boldsymbol{\Pi}_{1} \dot{\hat{\boldsymbol{\Upsilon}}}_{1}-\tilde{\boldsymbol{\Upsilon}}_{2}^{T} \boldsymbol{\Pi}_{2} \dot{\hat{\boldsymbol{\Upsilon}}}_{2}-\varphi_{m} \tilde{m} \dot{\hat{m}}
\end{aligned}
$$

where $\tilde{\boldsymbol{D}}=\boldsymbol{D}-\hat{\boldsymbol{D}}, \tilde{\boldsymbol{\Upsilon}}_{1}=\boldsymbol{\Upsilon}_{1}-\hat{\boldsymbol{\Upsilon}}_{1}, \tilde{\boldsymbol{\Upsilon}}_{2}=\boldsymbol{\Upsilon}_{2}-\hat{\boldsymbol{\Upsilon}}_{2}$, with $\hat{\boldsymbol{D}}=\left[\hat{d}_{i}\right] \in R^{5}, i=1, \ldots, 5, \hat{\boldsymbol{\Upsilon}}_{1}=\left[\hat{f}_{j 1}\right] \in R^{3}$, $\hat{\Upsilon}_{2}=\left[\hat{f}_{j 2}\right] \in R^{3}, j=1,2,3$.

Arranging the form of $\dot{V}$, it is that

$$
\begin{aligned}
\dot{V}= & \boldsymbol{S}^{T}\left(-\mathbf{I} \boldsymbol{S}-\mathbf{H} \operatorname{sgn}(\boldsymbol{S})-\boldsymbol{\Phi}(\boldsymbol{q})\left(\boldsymbol{v}_{1}(\dot{\boldsymbol{q}}) \tilde{\boldsymbol{\Upsilon}}_{1}\right.\right. \\
& \left.\left.+\boldsymbol{v}_{2}(\dot{\boldsymbol{q}}) \tilde{\boldsymbol{\Upsilon}}_{2}+\tilde{m} \boldsymbol{G}_{c}^{*}(\boldsymbol{q})\right)+\boldsymbol{M}^{-1} \tilde{\boldsymbol{D}}\right) \\
& -\tilde{\boldsymbol{D}}^{T} \boldsymbol{\Pi}_{d} \dot{\hat{\boldsymbol{D}}}-\tilde{\boldsymbol{\Upsilon}}_{1}^{T} \boldsymbol{\Pi}_{1} \dot{\hat{\boldsymbol{\Upsilon}}}_{1}-\tilde{\boldsymbol{\Upsilon}}_{2}^{T} \boldsymbol{\Pi}_{2} \dot{\hat{\boldsymbol{\Upsilon}}}_{2}-\varphi_{m} \tilde{m} \dot{\hat{m}} \\
= & \boldsymbol{S}^{T}(-\mathbf{I} \boldsymbol{S}-\boldsymbol{H} \operatorname{sgn}(\boldsymbol{S}))-\tilde{\boldsymbol{\Upsilon}}_{1}^{T}\left(\boldsymbol{S}^{T} \boldsymbol{\Phi}(\boldsymbol{q}) \boldsymbol{v}_{1}(\dot{\boldsymbol{q}})\right)^{T} \\
& -\tilde{\boldsymbol{\Upsilon}}_{2}^{T}\left(\boldsymbol{S}^{T} \boldsymbol{\Phi}(\boldsymbol{q}) \boldsymbol{v}_{2}(\dot{\boldsymbol{q}})\right)^{T}-\tilde{m} \boldsymbol{S}^{T} \boldsymbol{\Phi}(\boldsymbol{q}) \boldsymbol{G}_{c}^{*}(\boldsymbol{q}) \\
& +\tilde{\boldsymbol{D}}^{T}\left(\boldsymbol{S}^{T} \boldsymbol{M}^{-1}\right)^{T}-\tilde{\boldsymbol{D}}^{T} \boldsymbol{\Pi}_{d} \dot{\hat{\boldsymbol{D}}}
\end{aligned}
$$

$$
\begin{aligned}
& -\tilde{\boldsymbol{\Upsilon}}_{1}^{T} \boldsymbol{\Pi}_{1} \dot{\hat{\boldsymbol{\Upsilon}}}_{1}-\tilde{\boldsymbol{\Upsilon}}_{2}^{T} \boldsymbol{\Pi}_{2} \dot{\hat{\boldsymbol{\Upsilon}}}_{2}-\varphi_{m} \tilde{m} \dot{\hat{m}} \\
= & \boldsymbol{S}^{T}(-\mathbf{I} \boldsymbol{S}-\mathbf{H} \operatorname{sgn}(\boldsymbol{S})) \\
& -\tilde{\boldsymbol{\Upsilon}}_{1}^{T}\left(\left(\boldsymbol{S}^{T} \boldsymbol{\Phi}(\boldsymbol{q}) \boldsymbol{v}_{1}(\dot{\boldsymbol{q}})\right)^{T}+\boldsymbol{\Pi}_{1} \dot{\hat{\boldsymbol{\Upsilon}}}_{1}\right) \\
& -\tilde{\boldsymbol{\Upsilon}}_{2}^{T}\left(\left(\boldsymbol{S}^{T} \boldsymbol{\Phi}(\boldsymbol{q}) \boldsymbol{v}_{2}(\dot{\boldsymbol{q}})\right)^{T}+\boldsymbol{\Pi}_{2} \dot{\hat{\boldsymbol{\Upsilon}}}_{2}\right) \\
& +\tilde{\boldsymbol{D}}^{T}\left(\left(\boldsymbol{S}^{T} \boldsymbol{M}^{-1}\right)^{T}-\boldsymbol{\Pi}_{d} \dot{\hat{\boldsymbol{D}}}\right) \\
& -\tilde{m}\left(\boldsymbol{S}^{T} \boldsymbol{\Phi}(\boldsymbol{q}) \boldsymbol{G}_{c}^{*}(\boldsymbol{q})+\varphi_{m} \dot{\hat{m}}\right) .
\end{aligned}
$$

Then, substituting (28) - (30) into the above equation, the result is as follows:

$\dot{V}=-\boldsymbol{S}^{T} \mathbf{I} \boldsymbol{S}-\boldsymbol{S}^{T} \mathbf{H} \operatorname{sgn}(\boldsymbol{S}) \leq 0$.

From (36) and (33), it is implied that $\boldsymbol{S} \in L_{\infty}$, and combining (15), yields, $\dot{\boldsymbol{S}} \in L_{\infty}$. To further prove it, the following invariant and compact set $\Xi$ with its largest invariant set $\Omega$ is utilized:

$\Xi=\left\{\left(\boldsymbol{S}\left(\boldsymbol{q}, \dot{\boldsymbol{q}}, \boldsymbol{q}_{r}, \dot{\boldsymbol{q}}_{r}\right), \dot{\boldsymbol{S}}\left(\boldsymbol{q}, \dot{\boldsymbol{q}}, \boldsymbol{q}_{r}, \dot{\boldsymbol{q}}_{r}\right) \mid \dot{V}=0\right\}\right.$.

In $\Omega$, the following relations $\boldsymbol{S}=0, \dot{\boldsymbol{S}}=0$ are derived. Thus, combining (14) and (17), the following equations are correct:

$$
\begin{aligned}
& \dot{\boldsymbol{e}}+\boldsymbol{\Lambda} \hat{\boldsymbol{\Delta}}(t) \boldsymbol{e}=0, \\
& \ddot{\boldsymbol{e}}+\boldsymbol{\Lambda} \dot{\hat{\Delta}}(t) \boldsymbol{e}+\boldsymbol{\Lambda} \hat{\boldsymbol{\Delta}}(t) \dot{\boldsymbol{e}}=0 .
\end{aligned}
$$

From (38), we have

$\lim _{t \rightarrow \infty} \boldsymbol{e}=0, \lim _{t \rightarrow \infty} \dot{e}=0$,

then, the conclusions in (39) and (40) produce $\lim _{t \rightarrow \infty} \ddot{e}=$ 0 .

Considering (5), the following relationship equations about reference trajectories are obvious,

$$
\begin{gathered}
\lim _{t \rightarrow \infty} \alpha=\alpha_{d}, \lim _{t \rightarrow \infty} x=x_{d}, \lim _{t \rightarrow \infty} l=l_{d}, \\
\lim _{t \rightarrow \infty} \dot{\alpha}=0, \lim _{t \rightarrow \infty} \dot{x}=0, \lim _{t \rightarrow \infty} \dot{l}=0, \\
\lim _{t \rightarrow \infty} \ddot{\alpha}=0, \lim _{t \rightarrow \infty} \ddot{x}=0, \lim _{t \rightarrow \infty} \ddot{l}=0 .
\end{gathered}
$$

Besides, from Eqs. (33) and (36), we can infer that $\lim _{t \rightarrow \infty} \tilde{f}_{i j}=0, i=1,2,3, j=1,2, \lim _{t \rightarrow \infty} \tilde{d}_{k}=0, k=$ $1, \ldots, 5, \lim _{t \rightarrow \infty} \tilde{m}=0$, which means the estimated parameters are consistent to the real based on Lyapunov stability.

Therefore, through the above analysis, the control system state is satisfied the following equation:

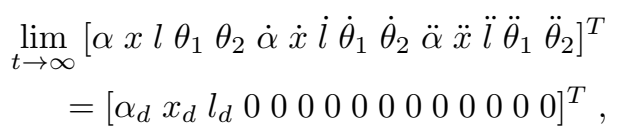

which distinctly means that $\Omega$ only includes the desired equilibrium point of the tower cranes closed-loop control system. Hence, Theorem 1 is completely proved under Lyapunov technique and LaSalle's invariance principle [33]. 
Table 1 Tower crane model parameters

\begin{tabular}{llll}
\hline$M_{t}[\mathrm{~kg}]$ & $m[\mathrm{~kg}]$ & $J_{0}\left[\mathrm{~kg} \cdot \mathrm{m}^{2}\right]$ & $g\left[\mathrm{~m} / \mathrm{s}^{2}\right]$ \\
\hline 3.6 & 1.0 & 6.8 & 9.8 \\
$\alpha_{0}[\mathrm{deg}]$ & $x_{0}[\mathrm{~m}]$ & $l_{0}[\mathrm{~m}]$ & - \\
15.0 & 0.3 & 0.5 & - \\
\hline
\end{tabular}

Table 2 Quantified analysis data

\begin{tabular}{llll}
\hline Control method & $t_{\alpha r}[\mathrm{~s}]$ & $t_{x r}[\mathrm{~s}]$ & $t_{l r}[\mathrm{~s}]$ \\
\hline Proposed method & 4.78 & 5.07 & 4.77 \\
SMC method & 5.61 & 6.01 & 4.79 \\
LQR method & 16.31 & 13.43 & 7.03 \\
& $\theta_{1 \max }[\mathrm{deg}]$ & $\theta_{2 \max }[\mathrm{deg}]$ & - \\
Proposed method & 0.69 & 0.33 & - \\
SMC method & 1.47 & 0.68 & - \\
LQR method & 2.10 & 1.09 & - \\
\hline
\end{tabular}

\section{Simulation Results and Discussion}

In this section, MATLAB\&Simulink was used, which verified the control effectiveness and strong robustness of the proposed method. First of all, to compare with the proposed method, a SMC method and an LQR method were designed and applied to the tower crane. Then, the extremely strong robustness of the proposed method was convincingly verified aiming at external disturbances of every DOF and model parameters uncertainties.

\subsection{Simulation Conditions}

The model parameters of the tower crane in simulations are given in Table 1, and the controller parameters of the proposed method were presented as follows:

$$
\begin{aligned}
& k_{1}=0.19, k_{2}=0.1, k_{3}=5.5, k_{4}=7.8, k_{5}=7.8, \\
& \mu_{1}=\mu_{2}=\mu_{3}=\mu_{4}=\mu_{5}=280, \\
& \gamma_{1}=\gamma_{2}=\gamma_{3}=\gamma_{4}=\gamma_{5}=80, \\
& \varphi_{11}=0.01, \varphi_{12}=0.01, \varphi_{13}=0.3, \\
& \varphi_{21}=0.1, \varphi_{22}=0.1, \varphi_{23}=0.5, \\
& \varphi_{d 1}=0.5, \varphi_{d 2}=0.5, \varphi_{d 3}=0.5, \\
& \varphi_{d 4}=0.0005, \varphi_{d 5}=0.0005, \\
& \varepsilon=0.5 .
\end{aligned}
$$

In addition, the reference trajectories parameters (i.e., $\left.\alpha_{d}, x_{d}, l_{d}, \alpha_{0}, x_{0}, l_{0}, t_{d}\right)$ were respectively set as
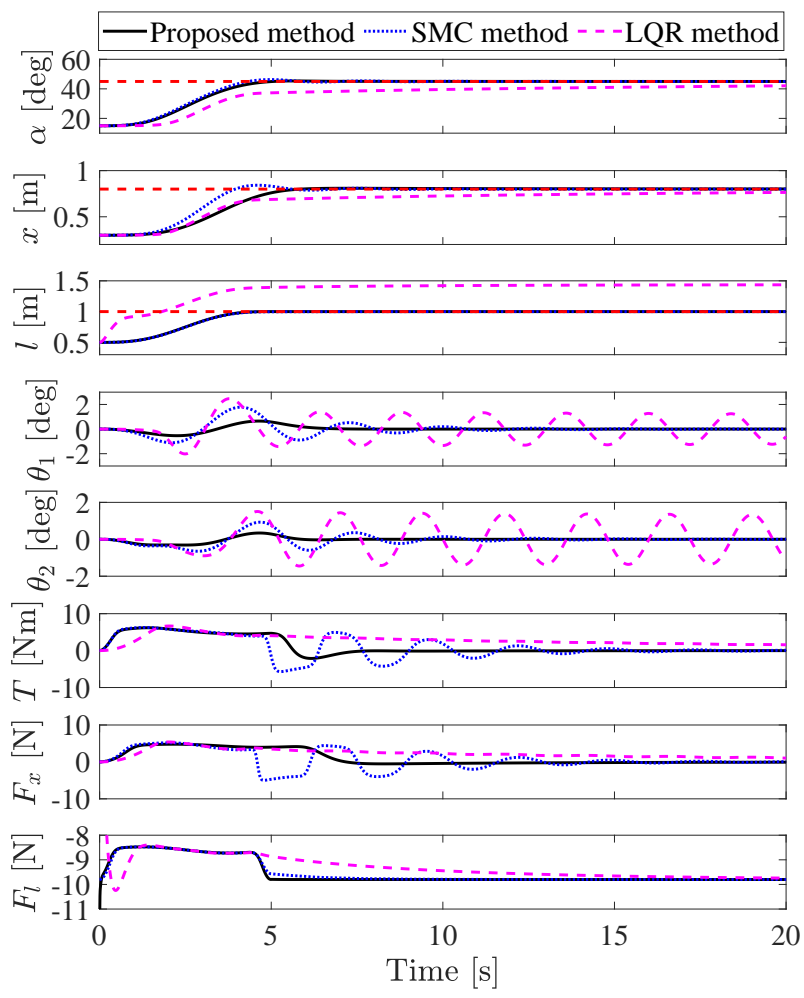

Fig. 2 Comparative simulation results

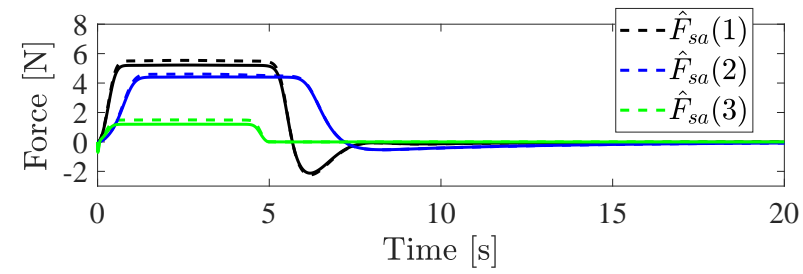

Fig. 3 Simulation results of estimation of friction

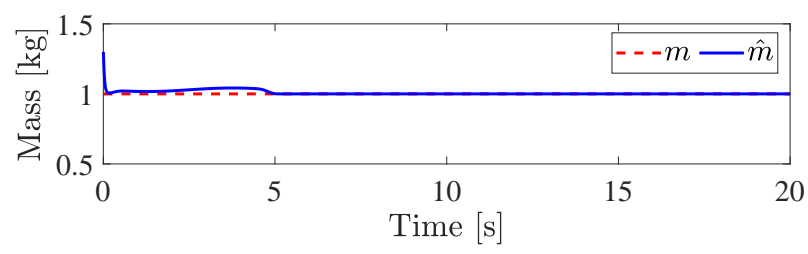

Fig. 4 Simulation results of estimation of $m$

$45.0[\mathrm{deg}], 0.8[\mathrm{~m}], 1.0[\mathrm{~m}], 15.0[\mathrm{deg}], 0.3[\mathrm{~m}], 0.5[\mathrm{~m}], 5[\mathrm{~s}]$, respectively. 


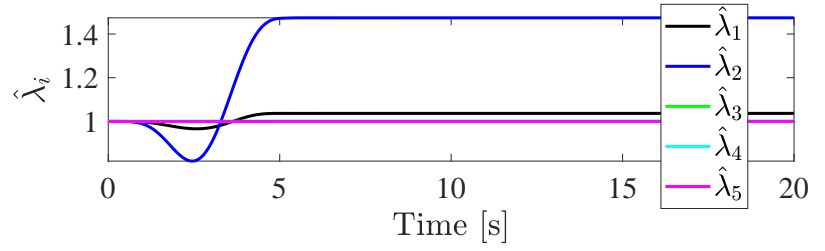

Fig. 5 Simulation results of $\lambda_{i}$

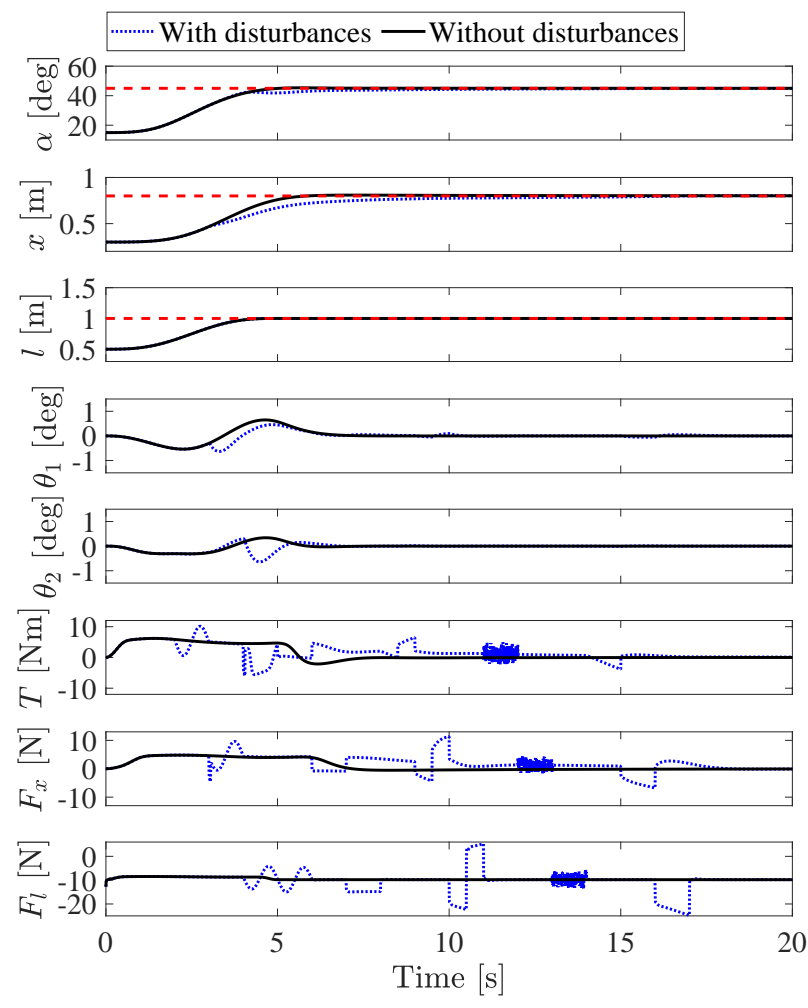

Fig. 6 Simulation results with external disturbances

\subsection{Comparative Simulations}

In this section, an SMC method and an LQR method were presented, which were compared with the proposed method.

The SMC method was introduced as follows:

$$
\begin{array}{r}
\boldsymbol{U}_{a}=\left(\boldsymbol{\Phi}(\boldsymbol{q})^{T} \boldsymbol{\Phi}(\boldsymbol{q})\right)^{-1} \boldsymbol{\Phi}(\boldsymbol{q})^{T}\left(-\boldsymbol{\Gamma}(\boldsymbol{q}, \dot{\boldsymbol{q}})+\ddot{\boldsymbol{q}}_{r}(t)\right. \\
-\boldsymbol{\Theta} \dot{\boldsymbol{e}}-\mathbf{I} \boldsymbol{S}-\mathbf{H} \tanh (\boldsymbol{S})),
\end{array}
$$

where $\boldsymbol{S}=\dot{\boldsymbol{e}}+\boldsymbol{\Theta} \boldsymbol{e}, \boldsymbol{\Theta}=\operatorname{diag}\left\{\varsigma_{1} \varsigma_{2} \varsigma_{3} \varsigma_{4} \varsigma_{5}\right\} \in R^{5 \times 5}$, and $\varsigma_{1}=0.8, \varsigma_{2}=0.8, \varsigma_{3}=0.9, \varsigma_{4}=2.5, \varsigma_{5}=2.9$.

To design the LQR method, the tower crane system model was linearized in equilibrium point firstly, and it was designed as follows:

$$
\begin{aligned}
& T=-k_{11} e_{\alpha}-k_{12} \dot{\boldsymbol{e}}_{\alpha}-k_{13} \theta_{2}-k_{14} \dot{\theta}_{2}, \\
& F_{x}=-k_{21} e_{x}-k_{22} \dot{\boldsymbol{e}}_{x}-k_{23} \theta_{1}-k_{24} \dot{\theta}_{1},
\end{aligned}
$$
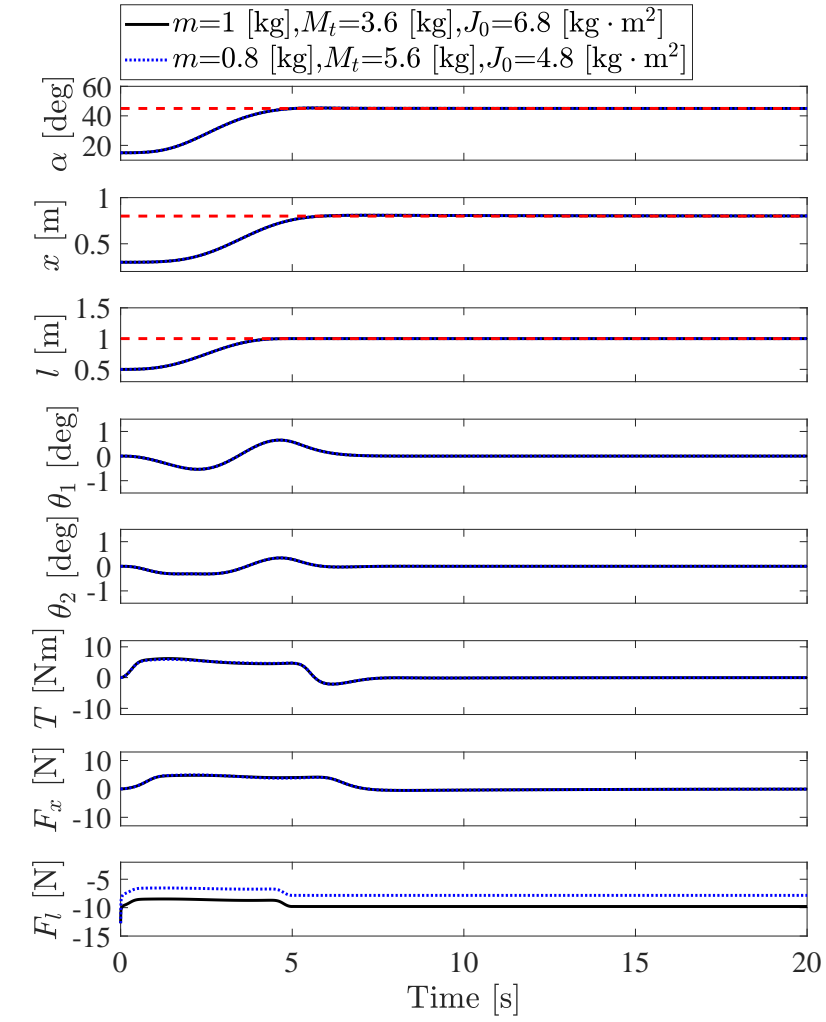

Fig. 7 Simulation results with model uncertainties

$F_{l}=-k_{31} e_{l}-k_{32} \dot{e}_{l}$,

where $\left[e_{\alpha}, \dot{e}_{\alpha}, e_{x}, \dot{e}_{x}, e_{l}, \dot{e}_{l}, \theta_{1}, \dot{\theta}_{1}, \theta_{2}, \dot{\theta}_{2}\right]^{T}$ was the system state vector,

and $Q=\operatorname{diag}\{1000,1,1000,1,500,1,5,1,5,1\}, \quad R=1$. Thus, the controller gains $k_{11}=31.62, k_{12}=24.89$, $k_{13}=-0.41, k_{14}=0.92, k_{21}=31.62, k_{22}=16.83$, $k_{23}=1.33, k_{24}=2.00, k_{31}=22.36, k_{32}=6.76$.

Remark 4: Traditional methods are unthoughtful about disturbances and model uncertainties, as shown in (46) and (47)-(49), where they all do not have the frictional compensation and disturbance suppression terms, and also, if the value of $m$ in the gravity compensation term is inaccurate, the control system easily loses stability.

In the comparative simulations, the disturbances were not considered, and the system only had friction condition. Figure 2 shows the comparative results, which contain the system state and controller outputs values. And meanwhile, the quantified control index data is $\mathrm{p}$ resented in Table 2 for the sake of comparison of control effects directly.

Firstly, in the positioning effect aspect, on account of friction, the LQR method was hard to accomplish it, and thus leaded to arrive target values after very long time. For rope length varying motion, because of gravity, the payload is left at a unexpected height by LQR, 
which is completely loss of control. However in the proposed method, it estimated the frictional force as shown in Fig. 3, thus, the control speed was fast in achieving positioning. And for $l$, the value of $m$ is estimated, where the result is in Fig. 4. Thus, the positioning for $l$ is stable. For the SMC method, it is obvious that its sliding mode surface gains matrix $\Theta$ was invariable, which would result in the control-related gains were difficult to tune and the convergence speed was inflexible, however in the proposed controller, $\lambda_{i}(t)$ was adaptive, as shown in Fig. 5. In Fig. 2, it can be found that the SMC method had evident overshoot in positioning and its outputs values were not close to zero until about 15 [s]. All in all, the proposed method was fast and did not have overshoot in positioning.

Then, the swing angle suppression results of the SM$\mathrm{C}$ and LQR method were pale by comparison. In Table 2 , it is shown that the maximum swing angles were smallest under the proposed controller. In Fig. 2, the LQR controller was almost impossible to suppress the swing angles, where the oscillation phenomenon kep$\mathrm{t}$ appearing. And, the convergence speed of the swing angles by using the SMC controller was slower than the proposed method.

\subsection{Robust Performance}

In this section, the strong robustness of the proposed method was verified in regard to disturbances of all D$\mathrm{OF}$ and model parameters uncertainties.

The external disturbances were added, which were asynchronous, unordered, various and intense. Even though under such disturbances, the positioning and swing suppression aspect almost had none negative impacts, which were benefited by the adaptive compensation. For the proposed controller, the compensation effect can be seen obviously in Fig. 6 where the controller outputs compensated the disturbance timely.

Different model parameters condition was considered, which is shown in Fig. 7. In this simulation, we changed the model parameters from that in Table 1 to $m=0.8[\mathrm{~kg}], M_{t}=5.6[\mathrm{~kg}]$ and $J_{0}=4.8\left[\mathrm{~kg} \cdot \mathrm{m}^{2}\right]$ but still used the model parameters in Table 1 for the proposed controller. It is found that the control effect did not have marked difference, and the adaptive part had work in compensating different $m$. Therefore, the proposed controller is insensitive to unknown model parameters.

\section{Conclusion}

In this paper, we presented a robust adaptive sliding mode controller for the tower crane with time-varying rope length, which has nonlinear adaptive sliding surface and adaptive compensation for frictions, disturbances and unknown payload mass to effectively realize the positioning of the jib, trolley and rope and meanwhile achieve swing elimination. It's worth noting that the controller was designed and analyzed without a linearized model. And at the same time, the nonzero initial reference trajectories were used, leading to the controller is more practical and safer, and also do not have big initial output values even though in large control gains cases. Lyapunov technique and LaSalle's invariance principle were detailed utilized to prove the control system stability theoretically. Sufficient simulations were applied, which showed that the tower crane control system is better and has strong robustness with respect to disturbances and model uncertainties under the proposed controller.

\section{Conflict of Interests}

The authors declare that there is no conflict of interests regarding the publication of this article.

\section{Acknowledgement}

This work is supported in part by the National Natural Science Foundation of China under Grants 51707092 and 61703202 .

\section{References}

1. W. Liu, S. Chen and H. Huang, "Double closed-loop integral terminal sliding mode for a class of underactuated systems based on sliding mode observer," International Journal of Control, Automation and Systems, vol. 18, pp. 339-350, 2020.

2. H. Yabuno and S. Kobayashi, "Motion control of a flexible underactuated manipulator using resonance in a flexible active arm," International Journal of Mechanical Sciences, vol. 174, p. 105432, 2020.

3. H. Yin, Y. Chen, J. Huang, and H. Lü, "Tackling mismatched uncertainty in robust constraint-following control of underactuated systems," Information Sciences, vol. 520, pp. 337-352, 2020.

4. O. D. Montoya and W. Gil-Gonzáez, "Nonlinear analysis and control of a reaction wheel pendulum: Lyapunovbased approach," Engineering Science and Technology, an International Journal, vol. 23, pp. 21-29, 2020.

5. S. Zhang, X. He, H. Zhu, Q. Chen, and Y. Feng, "Partially saturated coupled-dissipation control for underactuated overhead cranes," Mechanical Systems and Signal Processing, vol. 136, p. 106449, 2020. 
6. M. J. Maghsoudi, Z. Mohamed, A. R. Husain, and M. O. Tokhi, "An optimal performance control scheme for a 3D crane," Mechanical Systems and Signal Processing, vol. 66-67, pp. 756-768, 2016.

7. L. A. Tuan, "Fractional-order fast terminal back-stepping sliding mode control of crawler cranes," Mechanism and Machine Theory, vol. 137, pp. 297-314, 2019.

8. B. Lu, Y. Fang and N. Sun, "Sliding mode control for underactuated overhead cranes suffering from both matched and unmatched disturbances," Mechatronics, vol. 47, pp. 116-125, 2017.

9. H. Chen, Y. Fang and N. Sun, "An adaptive tracking control method with swing suppression for 4-DOF tower crane systems," Mechanical Systems and Signal Processing, vol. 123, pp. 426-442, 2019.

10. J. Gao, L. Wang, R. Gao, and J. Huang, "Adaptive control of uncertain underactuated cranes with a nonrecursive control scheme," Journal of the Franklin Institute, vol. 356, pp. 11305-11317, 2019.

11. M. Zhang, Y. Zhang, H. Ouyang, C. Ma, and X. Cheng, "Adaptive integral sliding mode control with payload sway reduction for 4-DOF tower crane systems," Nonlinear Dynamics, vol. 99, 2727-2741, 2020.

12. A. T. Le and S. Lee, "3D cooperative control of tower cranes using robust adaptive techniques," Journal of the Franklin Institute, vol. 354, pp. 8333-8357, 2017.

13. H. Ouyang, J. Wang, G. Zhang, L. Mei, and X. Deng, "Novel adaptive hierarchical sliding mode control for trajectory tracking and load sway rejection in doublependulum overhead cranes," IEEE Access, vol. 7, pp. 10353-10361, 2019.

14. S. Zhang, X. He and Q. Chen, "Energy coupleddissipation control for 3-dimensional overhead cranes," Nonlinear Dynamics, vol. 99, pp. 2097-2107, 2020.

15. N. Sun, T. Yang, Y. Fang, B. Lu, and Y. Qian, "Nonlinear motion control of underactuated three-dimensional boom cranes with hardware experiments," IEEE Transactions on Industrial Informatics, vol. 14, pp. 887-897, 2018.

16. S. Zhang, X. He, H. Zhu, Q. Chen, and Y. Feng, "Partially saturated coupled-dissipation control for underactuated overhead cranes," Mechanical Systems and Signal Processing, vol. 136, p. 106449, 2020.

17. T. D. Ho and K. Terashima, "Robust control designs of payload's skew rotation in a boom crane system," IEEE Transactions on Control Systems Technology, vol. 27, pp. 1608-1621, 2019.

18. X. Wu, K. Xu and X. He, "Disturbance-observer-based nonlinear control for overhead cranes subject to uncertain disturbances," Mechanical Systems and Signal Processing, vol. 139, p. 106631, 2020.

19. I. Golovin and S. Palis, "Robust control for active damping of elastic gantry crane vibrations," Mechanical Systems and Signal Processing, vol. 121, pp. 264-278, 2019.

20. B. Kolar, H. Rams and K. Schlacher, "Time-optimal flatness based control of a gantry crane," Control Engineering Practice, vol. 60, pp. 18-27, 2017.

21. M. Hamdy, R. Shalaby and M. Sallam, "Experimental verification of a hybrid control scheme with chaotic whale optimization algorithm for nonlinear gantry crane: A comparative study," ISA Transactions, vol. 98, pp. 418433, 2020.

22. L. Rincon, Y. Kubota, G. Venture, and Y. Tagawa, "Inverse dynamic control via "simulation of feedback control" by artificial neural networks for a crane system," Control Engineering Practice, vol. 94, p. 104203, 2020.

23. L. A. Tuan, H. M. Cuong, P. V. Trieu, L. C. Nho, V. D. Thuan, and L. V. Anh, "Adaptive neural network sliding mode control of shipboard container cranes considering actuator backlash," Mechanical Systems and Signal Processing, vol. 112, pp. 233-250, 2018.

24. L. Ramli, Z. Mohamed, M. Efe, I. M. Lazim, and H. I. Jaafar, "Efficient swing control of an overhead crane with simultaneous payload hoisting and external disturbances," Mechanical Systems and Signal Processing, vol. 135, p. 106326, 2020.

25. X. Li, X. Peng and Z. Geng, "Anti-swing control for 2D under-actuated cranes with load hoisting/lowering: A coupling-based approach," ISA Transactions, vol. 95, pp. 372-378, 2019.

26. Z. Zhang, Z. Zhang, Y. Wu, Y. Wu, J. Huang, and J. Huang, "Differential-flatness-based finite-time anti-swing control of underactuated crane systems," Nonlinear Dynamics, vol. 87, pp. 1749-1761, 2017.

27. N. Sun, Y. Fang, H. Chen, and B. He, "Adaptive nonlinear crane control with load hoisting/lowering and unknown parameters: design and experiments," IEEE/ASME Transactions on Mechatronics, vol. 20, pp. 2107-2119, 2015.

28. B. Lu, Y. Fang and N. Sun, "Enhanced-coupling adaptive control for double-pendulum overhead cranes with payload hoisting and lowering," Automatica, vol. 101, pp. 241-251, 2019 .

29. M. J. Maghsoudi, L. Ramli, S. Sudin, Z. Mohamed, A. R. Husain, and H. Wahid, "Improved unity magnitude input shaping scheme for sway control of an underactuated 3D overhead crane with hoisting," Mechanical Systems and Signal Processing, vol. 123, pp. 466-482, 2019.

30. T. Yang, N. Sun, H. Chen, and Y. Fang, "Observer-Based Nonlinear Control for Tower Cranes Suffering From Uncertain Friction and Actuator Constraints with Experimental Verification," IEEE Transactions on Industrial Electronics, p. 1-1, 2020.

31. N. Sun, Y. Wu, H. Chen, and Y. Fang, "Antiswing cargo transportation of underactuated tower crane systems by a nonlinear controller embedded with an integral term," IEEE Transactions on Automation Science and Engineering, vol. 16, pp. 1387-1398, 2019.

32. X. Zhou and X. Li, "A finite-time robust adaptive sliding mode control for electro-optical targeting system with friction compensation," IEEE Access, vol. 7, pp. 166318166328, 2019.

33. H. K. Khalil, "Nonlinear Systems", 3rd ed., Englewood Cliffs, NJ, USA: Prentice-Hall, 2002. 
Figures

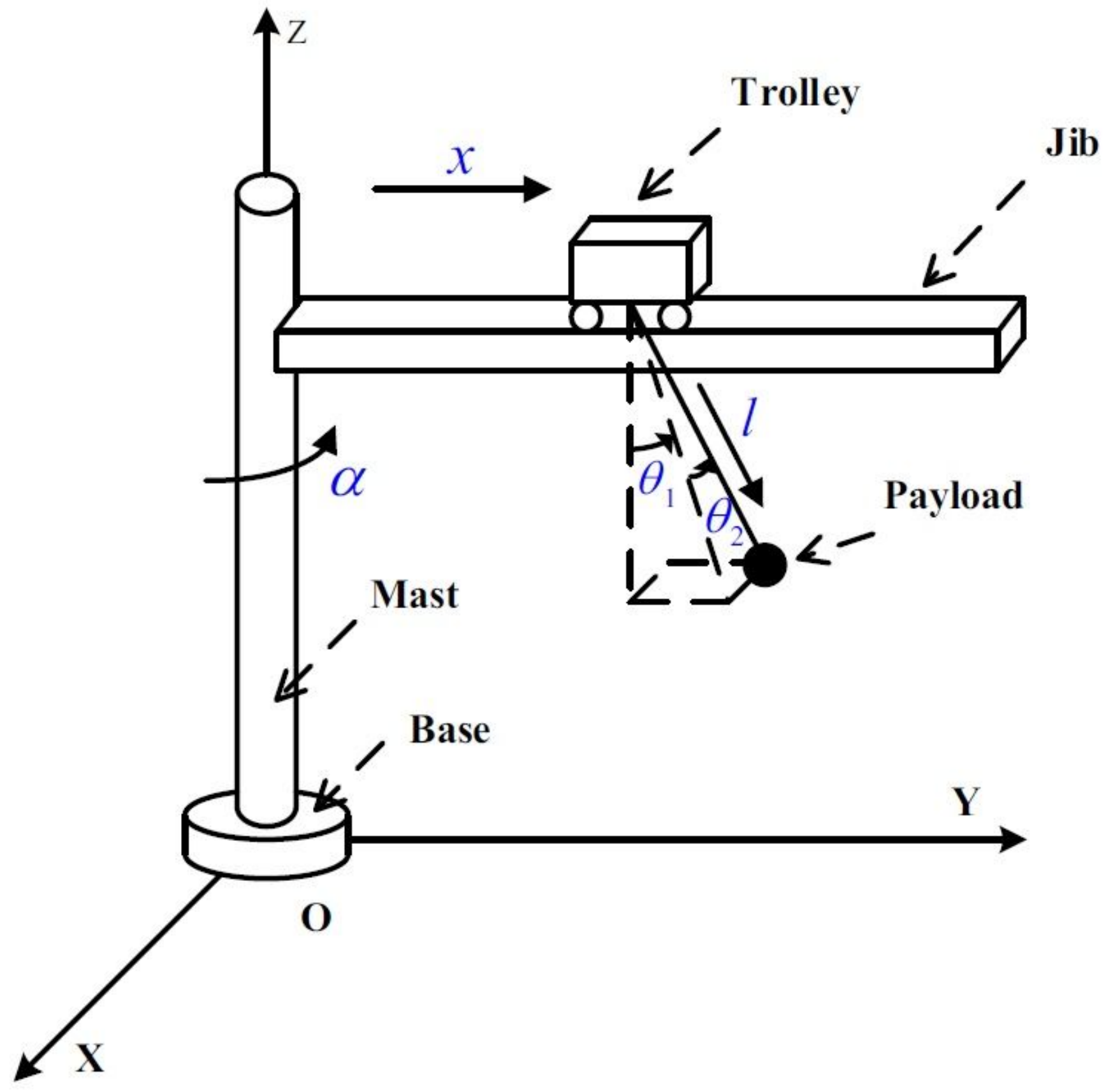

Figure 1

Tower crane model 

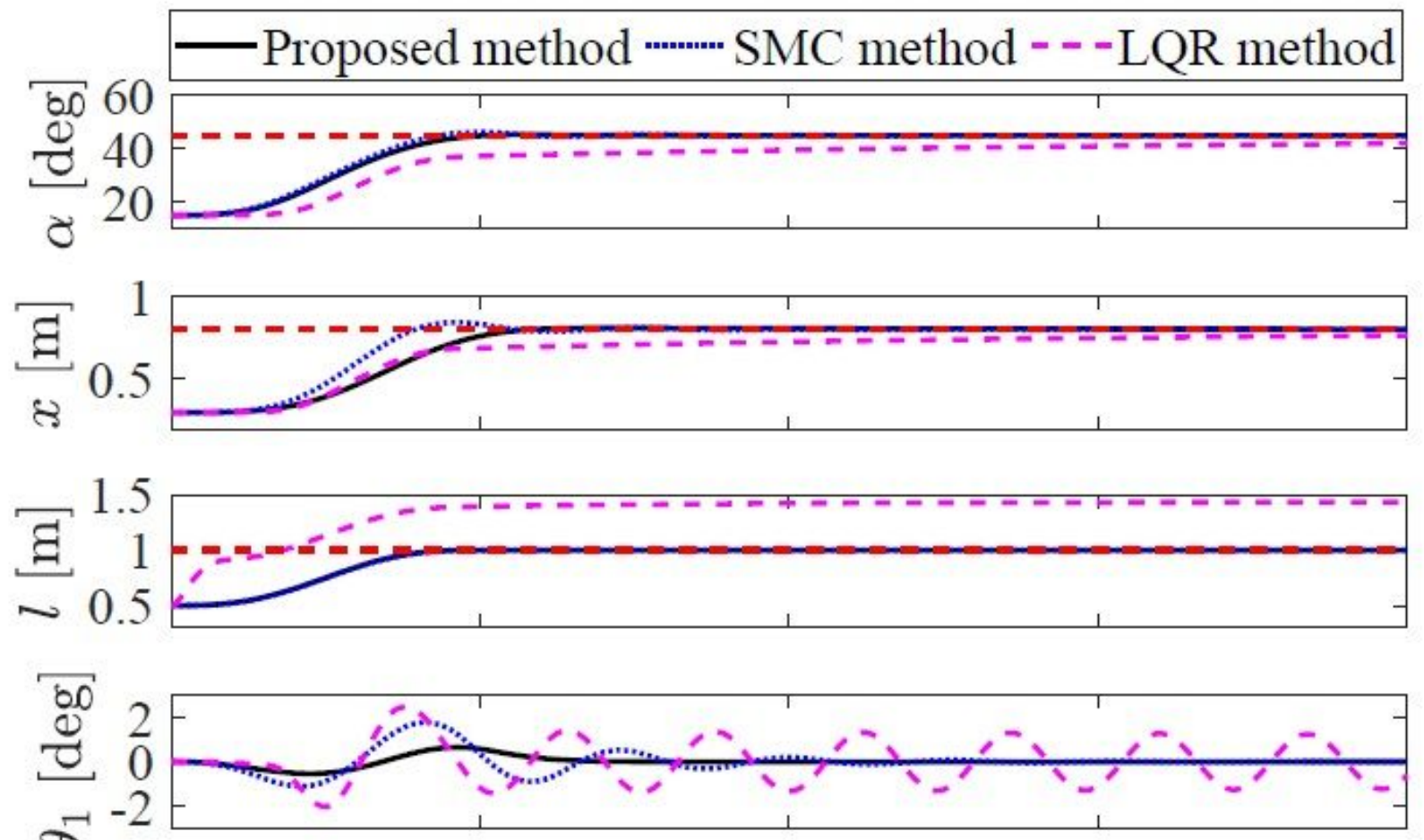

$\infty$
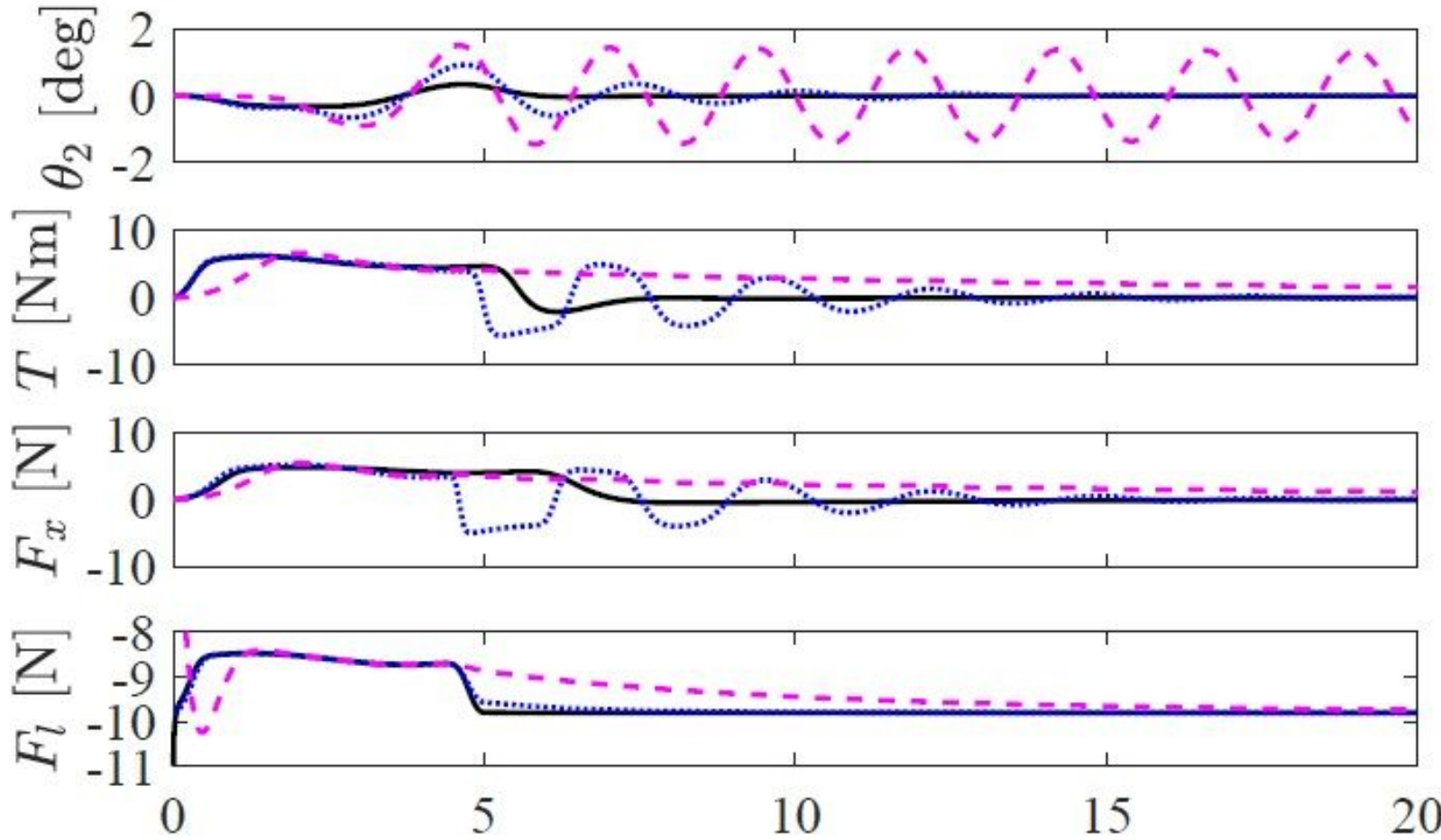

Time $[\mathrm{s}]$

Figure 2

Comparative simulation results 


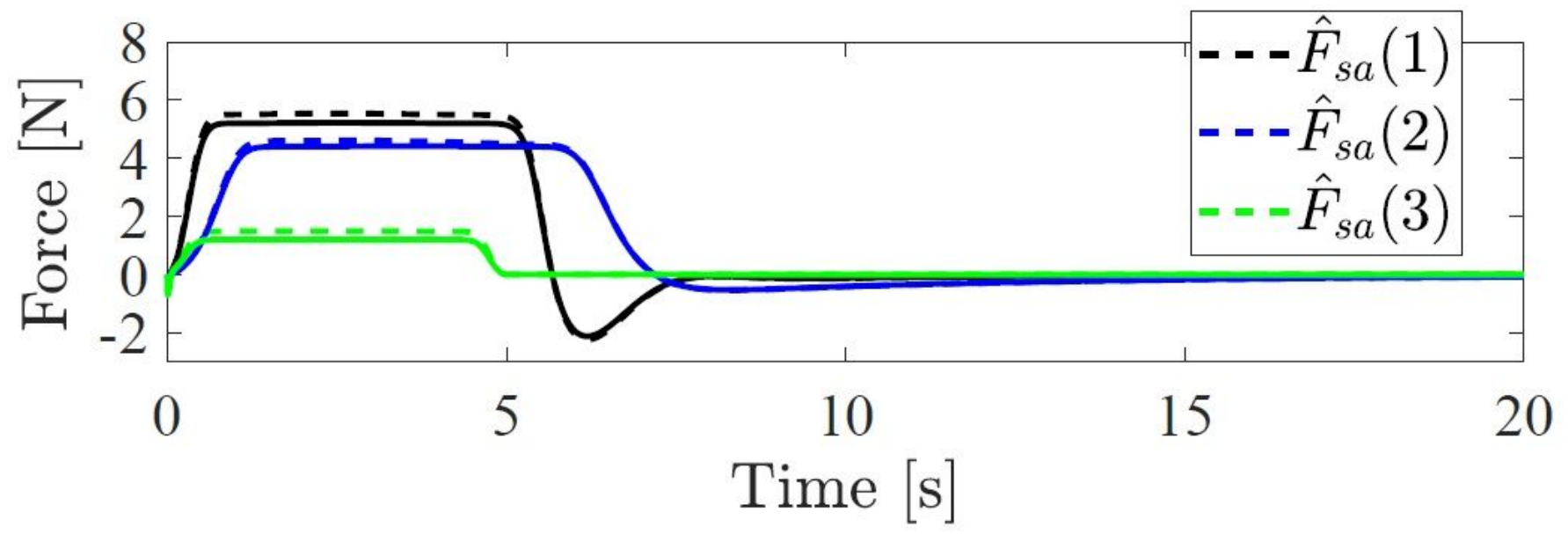

Figure 3

Simulation results of estimation of friction

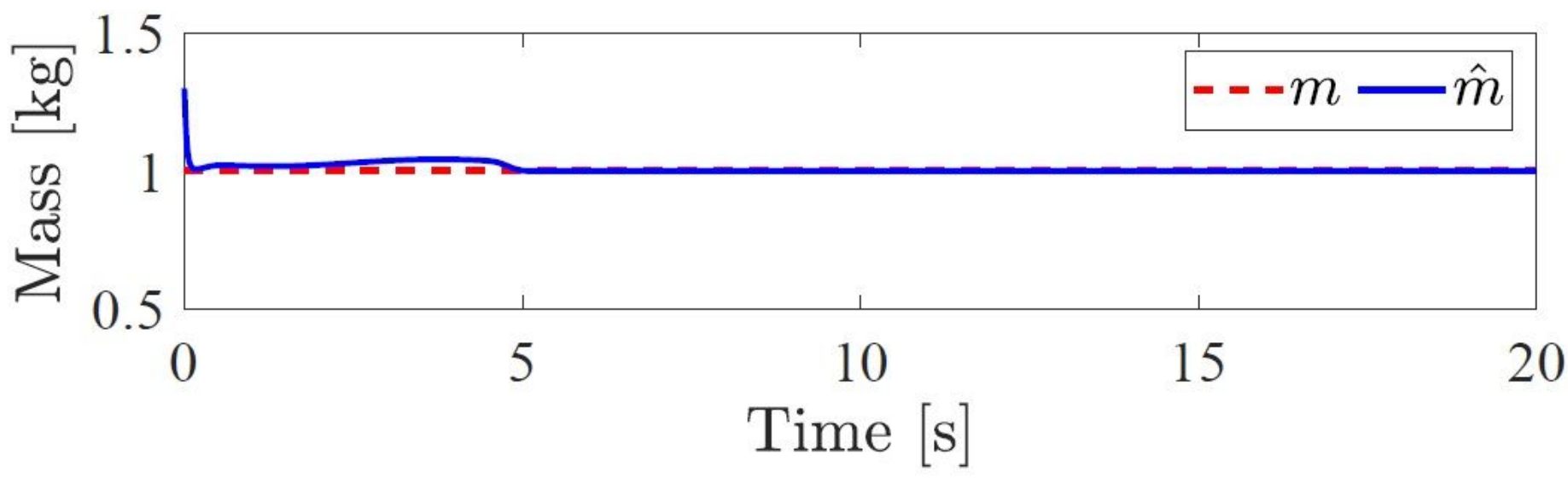

Figure 4

Simulation results of estimation of $\mathrm{m}$

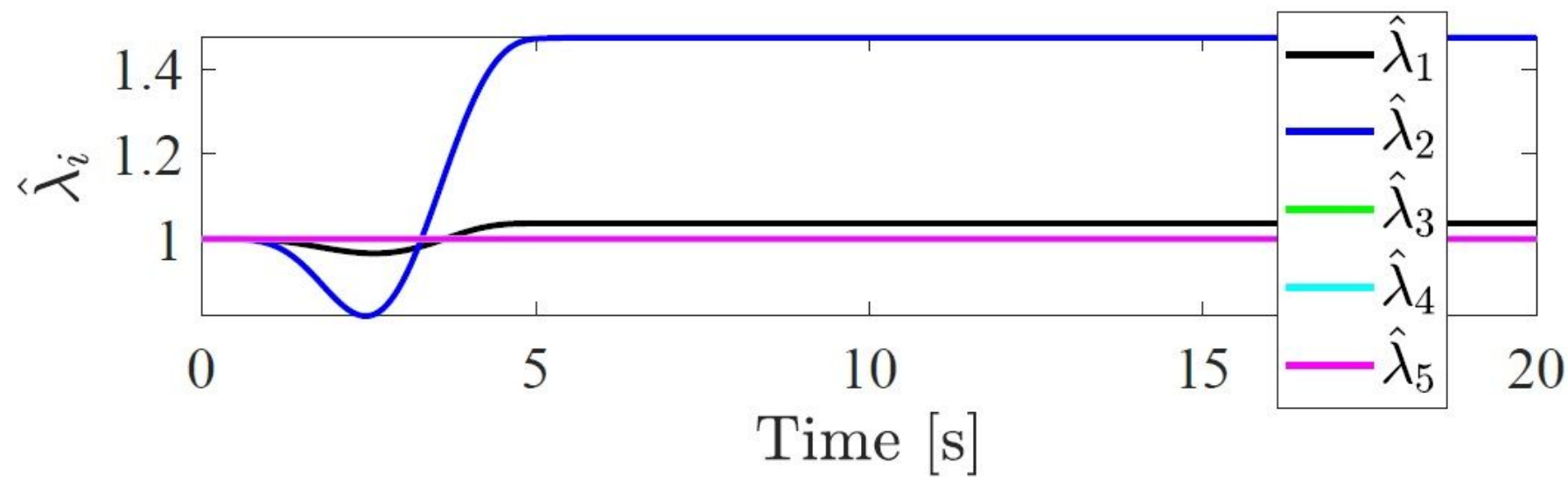

Figure 5 
Simulation results
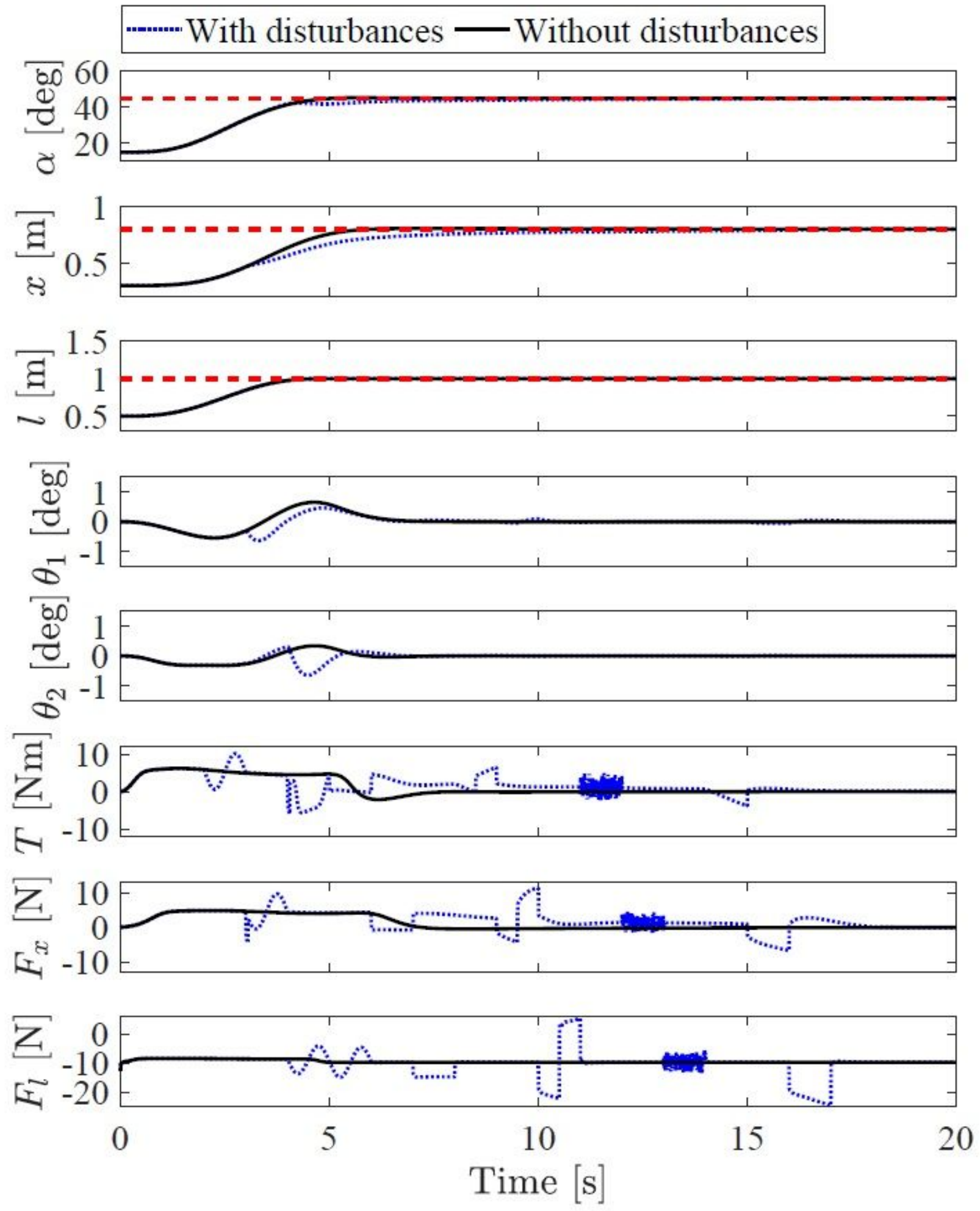

Figure 6

Simulation results with external disturbances 

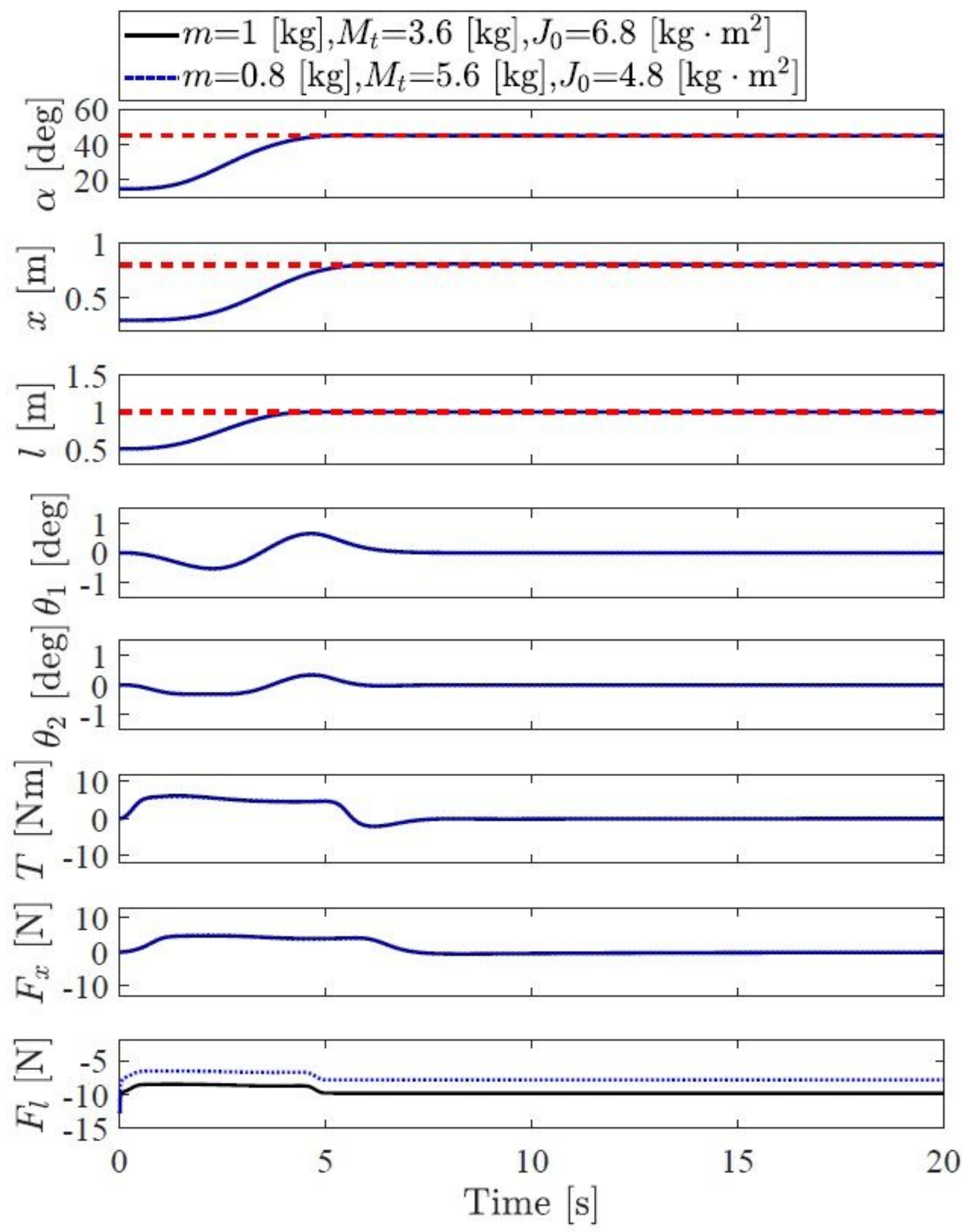

Figure 7

Simulation results with model uncertainties 